\title{
Albertus Magnus über die ars de symbolica theologia des Dionysius Areopagita ${ }^{1}$
}

\author{
Henryk Anzulewicz. \\ ALBERTUS-MAGNUS-INSTITUT, BONN
}

Über die Kenntnisse des Corpus Dionysiacum verfügte Albertus Magnus schon bei der Abfassung seines Erstlingswerkes De natura boni, das er in den 1230er Jahren als Lektor an den Konventen des Predigerordens der Provinz Teutonia vor seinem Studium und seiner Lehrtätigkeit an der Pariser Universität begann, wohl aber nicht vollendete. ${ }^{2}$ Die Schriften des historisch nicht identifizierbaren Autors, der sich für den Schüler des Apostel Paulus, Dionysius Areopagita, ausgab, benutzte der Doctor universalis reichlich in seinem theologiesystematischen Frühwerk, das vor der Kommentierung der Sententiae des Petrus Lombardus (begonnen um 1243 in Paris) entstanden ist. ${ }^{3}$ Diese theologisch profunde und wirksame, die Autorität des Apostelschülers für sich noch lange unangefochten beanspruchende und die Philosophie des Proklos christianisierende Quelle (einschließlich der darin überlieferten Scholien) wurde von Albert sodann

1 Diese Untersuchung ist ein Teil des Forschungsprojektes "El Misterio del Ser en Hans Urs von Balthasar, Albertus Magnus y Nicolás de Cusa", das vom Fondo Nacional de Desarrollo Científico y Tecnológico (FONDECYT, Santiago-Chile) Nr. 1100043 gefördert und von Frau Prof. Dr. Anneliese Meis, Pontificia Universidad Católica de Chile, Facultad de Teología, geleitet wird.

2 Albertus Magnus, De natura boni, ed. Ephrem Filthaut (Alberti Magni Opera Omnia XXV/1), Münster 1974.

3 Zur Echtheit des Corpus und seinem unbekannten Autor, dem vermeintlichen Dionysius Areopagita cf. Makris (2000), 3-39; Kapriev (2005), 41-44. Albertus hat in seiner Frühschrift De IV coaequaevis (Summa de creaturis I) das Werk De caelesti bierarchia des Dionysius seiner Engellehre zugrunde gelegt; sein Lehrstück stellt über weite Strecken einen Quästionenkommentar des Dionysischen Werkes dar. 
im Sentenzenkommentar oftmals herangezogen. ${ }^{4}$ Unser Autor reflektierte aber hierbei weder das Theologieverständnis des Areopagiten noch dessen Auffassung der symbolischen und der mystischen Gotteserkenntnis. Erst in seinen chronologisch auf den Sentenzenkommentar folgenden Kommentaren zu den Schriften aus dem Corpus Dionysiacum hob er den eigentümlichen Duktus der Dionysischen Theologie hervor, versah sie mit einer wissenschaftstheoretischen Grundlage, erklärte und entfaltete sie in systematischer Hinsicht.

Im Folgenden gilt unser Augenmerk Alberts wissenschaftstheoretischer Reflexion, Auslegung und Entfaltung der symbolischen Theologie des Dionysius. Bevor wir sie in den Blick nehmen, gehen wir kurz auf die Frage ein, ob das dionysische Theologieverständnis die theologische Wissenschaftslehre Alberts, die er erstmalig im Sentenzenkommentar (Buch I) formulierte, und seine theologische Denkweise tatsächlich nicht schon früher beeinflusste.

Es steht fest, dass dem Denken des Albertus Magnus ein dezidiert theologisches, mit dem neuplatonischen Reflexionsmuster weitgehend konformes Strukturprinzip zugrunde liegt, das im Wesentlichen von der Denkweise des Dionysius (und vom Liber de causis) inspiriert ist. ${ }^{5}$ Dieser nachhaltige Einfluss des Areopagiten auf unseren Autor gab ihm

4 Zur Wirkmächtigkeit der Theologie des Dionysius im Mittelalter cf. Grabmann (1933), 23-24; zu den Gemeinsamkeiten und Unterschieden der Theologie des Dionysius und der neuplatonischen Philosophie des Proklos cf. Saffrey (1996), 218-219 und Beierwaltes (1997). Albert zitiert in seinem Sentenzenkommentar bereits bei der Erklärung des Prologs des Petrus Lombardus die Schriften des Dionysius mehrfach, cf. Albertus Magnus, In I Sententiarum, In prologum Magistri expositio, ed. Aug. Borgnet (Alberti Magni Opera Omnia XXV), Paris 1893, p.11a: "Unde Dionysius in libro De divinis nominibus: Fides est unicum credentium fundamentum, eos collocans in veritate, et in eis veritatem, incredibili incommutabilitate simplam veritatis scientiam habentibus credulis."; ibid. p.11a-b: "Unde Dionysius in Epistola ad Polycarpum Episc: Hoc quodcumque est secundum legem veritatis recte demonstrato et puro existente, omne quod aliter habet, et veritatem similat, reprehendetur, etc."; ibid. p.11b: "sicut dicit Commentator super primum caput De divinis nominibus: Nihil aliud est auctoritas quam rationis reperta veritas, ob posteritatis utilitatem scripto commendata. (...) sicut docet Dionysius in libro De divinis nominibus: Universaliter non audendum est dicere, nec etiam cogitare aliud de supersubstantiali et occulta divinitate, praeter ea quae divinitus nobis ex sacris eloquiis sunt expressa." Zum Verständnis der Bezeichnung 'Commentator' und den Scholien- und Glossen im Corpus Dionysiacum cf. Simon (1972), p.XVIII v.31-59; Suchla (21999), 175.

5 Anzulewicz (2000). Müller (2001), 368-371. Cf. Anzulewicz/Rigo (2002). 
den hermeneutischen Schlüssel für seine ganzheitliche Wirklichkeitsdeutung, die sich in seinen Werken widerspiegelt und die bis auf seine Erstlingsschrift De natura boni zurückverfolgt werden kann. ${ }^{6}$ Hingegen kam er in seinem Frühwerk, in dem er in Anlehnung an die Sententiae des Petrus Lombardus die Sakramentenlehre, die Christologie und die Eschatologie, sowie (von Lombarden nur themenspezifisch geleitet) die Schöpfungstheologie und die Tugendlehre behandelt hatte, nicht zur Darstellung der theologischen Wissenschaftslehre. Der Grund hierfür ist darin zu sehen, dass Albert in seinem Frühwerk die Themen, die mit den Inhalten der vier Bücher der Sententiae des Petrus Lombardus korrespondieren, in einer diesen gegenüber umgekehrten Reihenfolge (d. h. beginnend mit Themen von Buch IV) und nur jene behandelte, die zum Bestand der Sentenzenbücher IV, III und II gehörten. Er kam also im Frühwerk nicht bis zur Gotteslehre, die in Buch I der Sententiae enthalten war. In der Kommentierungstradition der Sententiae galt es aber inzwischen, dass die wissenschaftstheoretischen Einleitungsfragen der Darstellung der systematischen Theologie vorausgeschickt wurden. ${ }^{7}$ Dem vom Sentenzenmagister eingeführten Grundschema der inhaltlichen Strukturierung der Theologie zufolge bildete die Gotteslehre den heilsgeschichtlichen und doktrinellen Ausgangspunkt (und letztlich auch den Endpunkt) der Reflexion und somit den festen Bezugspunkt für die (vorgeschaltete) Verortung der wissenschaftstheoretischen Einleitungslehre in die Theologie. ${ }^{8}$

Bei der Ausarbeitung der theologischen Wissenschaftslehre zu Beginn seines Sentenzenkommentars (Buch I), die als die erste vollständige, kurz

6 Cf. Anzulewicz (2002); id. (2009).

7 Die Sententiae des Petrus Lombardus dienten spätestens seit dem IV. Laterankonzil (1215) als das Lehrbuch der christlichen, auf die Bibel und Kirchenväter gestützten Theologie und als Leitfaden und Vorlage für ihre systematische Darstellung. Die Konzepte der Wissenschaft und der Weisheit des Augustinus und des Aristoteles erörterte Albert zwar schon in der Abhandlung über den Cherubim im Kontext seiner Engellehre in der Frühschrift De IV coaequaevis (i. e. Summa de creaturis I, tr.4 q.38 a.1, ed. Steph. Caes. Aug. Borgnet, Alberti Magni Opera Omnia XXXIV, Paris 1895, p.549a-552b), aber er applizierte hierbei nur den Begriff der Weisheit auf die Theologie, während er den Innbegriff des vielschichtigen Aristotelischen Wissenschaftsverständnisses (scientia simpliciter, i. e. habitus intelligentiae speculativae) mit der Metaphysik gleichsetzte.

8 Zum zweifachen Strukturprinzip der Sententiae des Petrus Lombardus cf. Leinsle (1995), 93. 
gefasste Wissenschaftstheorie der Theologie überhaupt gilt, ${ }^{9}$ orientierte sich Albert am Wissenschaftsbegriff der Zweiten (und der Ersten) Analytiken des Aristoteles einschließlich diesbezüglicher Ergänzungen und Präzisierungen der arabischen Aristotelesinterpreten, insbesondere des Avicenna (Philosophia prima I 1). ${ }^{10}$ Er begründete die Theologie als Wissenschaft gemäß dem aristotelischen Wissenschaftsideal und grenzte sie wissenschaftstheoretisch von der Philosophie und den nichttheologischen Wissenschaftsdisziplinen ab, die er als von der Theologie unabhängige Wissenschaften je eigener Zuständigkeit und eigenen Rechts anerkannte. ${ }^{11}$ Hierbei ging er auf das dionysische Theologieverständnis und dessen Auffassung der symbolica theologia nicht ein.

Hinsichtlich der theologischen Methode unterschied Albert im Sentenzenkommentar zwischen der Auslegung (expositio) und der Beweisführung (probatio), zwischen zwei Vorgehensweisen, die er mit einer zweifachen Zielsetzung der Theologie als "Lehre" (doctrina) und als "Kunstfertigkeit" (ars), begründete. Als Ziel der methodischen Zweiteilung der Theologie bestimmte er zum einen die Unterweisung im rechten Glauben (exhortari in doctrina sana) und zum anderen die diskursivbeweisend verfahrende und die Irrlehren widerlegende Apologie und Vermittlung der Glaubenswahrheit (contradicentes revincere), die sich auf Autorität oder natürliche Vernunft stützten. In Bezug auf die Unterweisung, die erzieherisch-praktische Aufgabe der Theologie, erläuterte er u. a. die allegorische und die anagogische Methode der Schriftausle-

9 Den Entwicklungsgang der christlichen Theologie zur Wissenschaft vom 3. Jh. bis zu Pseudo-Dionysius Areopagita zeichnet mit einer scharfen Tiefenperspektive nach und unterstreicht die Bedeutung des Corpus Dionysiacum für die byzantinische und lateinische Theologie des 13. Jh. Henri D. Saffrey (1996), 201-220, bes. 218220. Für die weitere Entwicklung (bis Albertus Magnus) cf. Leinsle (1995), 16-151; Enders (2007), 19-37; speziell zu Albertus Magnus: Anzulewicz (2007); id. (im Druck I u. II).

10 Cf. Albertus Magnus, In I Sent. d.1 a.1, p.15a. Wichtige wissenschaftstheoretische Ansätze, insbesondere hinsichtlich der Fragen nach einer wissenschaftlichen Erkenntnis partikulärer Sachverhalte und der Notwendigkeit sowie des epistemischen Ranges solcher Wissenschaft fand Albert auch bei Averroes. Näheres hierzu cf. Anzulewicz (im Druck I u. II).

11 Alberts Neubestimmung des Verhältnisses von Theologie und Philosophie bedeutet eine epochale Zäsur in der Wissenschaftsgeschichte; cf. u. a. Wéber (1980), 559588; de Libera (1989), 49-67; Burger (1998), 579-586; Müller (2001), 24-61; Sicouly (2002), 23-37; id. (2003), 109-123; Anzulewicz (2007), 129-165; id. (2009), 219-221; Möhle (2008), 147-162; Senner (2009). 
gung, indes im Hinblick auf die 'diskursiv-beweisende Apologetik', die sich mit dem neuzeitlichen Begriff der systematischen Theologie deckt, hielt er fest, dass eine sachgerechte Argumentation sich auch passender Bilder (Analogien) bediene. ${ }^{12}$

Die im Sentenzenkommentar knapp dargelegte Auffassung der theologischen Wissenschaft im Modus der ars und die Zuordnung 'passend gewählter Ähnlichkeit' (similitudo congrue sumpta) als ihre Mittel bilden den Kern der symbolischen Theologie, die Albert nach der Fertigstellung des Sentenzenkommentars vor allem in seiner Auslegung des neunten Briefes aus dem Corpus Dionysiacum entfaltete. Wie er die symbolische Theologie des Dionysius interpretiert und wie er sich von seiner Textvorlage leiten lässt, aber auch inwiefern er über sie hinaus geht, ferner welche theologische (patristische und scholastische) und welche philosophische (antike und mittelalterliche) Autoritäten und Traditionen er integriert, soll hier anhand seines Kommentars zum neunten Brief des Dionysius skizziert werden. Parallelen aus Alberts Kommentar zu De mystica theologia und aus seiner Summa theologiae I, die zum besseren Verständnis oder zur Vertiefung von Alberts Interpretationen beitragen, werden gelegentlich berücksichtigt. Den Leitfaden dieser Skizze bildet jedoch, wie festgehalten, der Kommentar zu der Epistel des Areopagiten an einen dalmatinischen Bischof Titus, die nach Alberts Urteil ein vollständiges Kompendium der ars symbolicae theologiae darstellt. ${ }^{13}$

\section{II}

Mit seiner schon im Frühwerk dokumentierten Rezeption und anschlieBenden Kommentierung des Corpus Dionysiacum hat Albertus Magnus wesentlich dazu beigetragen, dass ein byzantinischer und für die lateinische Scholastik teilweise neuer, mit neuplatonischen Elementen durchsetzter Typus theologischer Reflexion - die symbolische, die kataphatische und die apophatische, $\mathrm{d}$. h. die affirmative und die negative, die diskursive und die mystische Theologie, besser bekannt und auch heimisch wurde. Diese Tatsache ist auf dem Hintergrund anfänglicher Assimilationsschwierigkeiten und doktrineller Gefahren, die man im 13. Jh. an der Pariser Universität im Werk Periphyseon des Dionysius-Übersetzers und produktiven

12 Albertus Magnus, In I Sent. d.1 a.5, p. 19b-20a.

13 Albertus Magnus, Super Dionysii Epistulas, ep. IX, ed. Paul Simon (Alberti Magni Opera Omnia XXXVII/2), Münster 1978, p.528 v.2-15. 
Vermittlers seines Denkens, Johannes Scotus Eriugena, zu erkennen und ihnen mit Zensuren und Verurteilung des Werkes zu begegnen vermochte, umso bedeutsamer. ${ }^{14}$

Wichtig ist ebenso die Tatsache, dass Albert die symbolische Theologie des Dionysius, über die der Areopagite nach eigener Bekundung ein Werk verfasst hatte, das aber im Corpus seiner Schriften nicht überliefert und wahrscheinlich nie geschrieben wurde,${ }^{15}$ in seinen Kommentaren, insbesondere zu De mystica theologia, nicht aus den Augen verlor und sie auf der Grundlage des neunten Briefes des Dionysius rekonstruierte, wissenschaftstheoretisch reflektierte und systematisch entfaltete. Der als ein Antwortschreiben an den Bischof Titus konzipierte Text beinhalte, schreibt er im Prolog des Kommentars, eine konzise Gesamtdarstellung der symbolischen Theologie, die er als die Kunstfertigkeit (ars) bezeichnet. Seine Auslegung der Ausführungen des Areopagiten zu den Fragen des Titus nach dem wahren Ort der Weisheit, der wahren Nahrung und des Brotes ${ }^{16}$ nimmt Albert zum Anlass, auch seine eigene Auffassung der symbolischen Theologie zu entfalten. Er beginnt in der für seine Werkkommentare (nicht aber für die Auslegung der übrigen Briefe des Dionysius) gewohnte Weise mit der wissenschaftstheoretischen Grundlegung der symbolischen Theologie und erklärt ihren Gegenstand -die Vielfalt

14 Zur Gedankenwelt des Eriugena cf. Beierwaltes (1994), mit Anknüpfung an Albertus Magnus, ebd. p.119. Zu den Zensuren und Verurteilung cf. Dondaine (1952), 73; Lucentini (1987); ferner Koch (1956-1957; Repr. 1973), 128-129 (Repr. 19-20).

15 Cf. Dionysius Areopagita, Epistula nona, in: Albertus Magnus, Super Dionysii Epistulas, ep. IX, p.528 v.70. Johannes Sarracenus beklagt im Prolog zu seiner Übersetzung von De mystica theologia, dass er trotz intensiver Suche in Griechenland nach diesem dionysischen Werk, es nicht gefunden hatte; cf. Albertus Magnus, Super Dionysii Mysticam theologiam, ed. Paul Simon (Alberti Magni Opera Omnia XXXVII/2), Münster 1978, p. 453 v.54-56; ibid. p.530 v.35-36; id., Super Dionysium De divinis nominibus, ed. Paul Simon (Alberti Magni Opera Omna XXXVII/1) Münster 1972, p.42 v.49-51, p.384 v.63-65. Suchla (1998), 174.

16 Die genannten Fragen des Titus an Dionysius, die gleichsam als die Überschrift des Briefes fungieren, erscheinen in der Druckausgabe des griechischen Textes von Jacques-Paul Migne (PG 3, 1104) und in den Dionysiaca (I, 624) sowie in der lateinischen Übersetzung von Hilduin und Johannes Scotus Eriugena, nicht aber in der kritischen Edition und in der Übersetzung des Johannes Sarracenus. Albert legt seinem Kommentar die lateinische Übersetzung des Johannes Sarracenus zugrunde. Cf. PseudoDionysius Areopagita, Epistula 9, ed. Adolf M. Ritter (Corpus Dionysiacum II. Patristische Studien und Texte 36), Berlin/New York 1991, 193. Albertus Magnus, Super Dionysii Epistulas, ep. IX, p.528 v.68-69 (mit diesbezügl. Anm. im Variantenapparat). 
von Metaphern und Symbolen-, ihre Eigenart und ihre Methoden sowie die inhaltliche Struktur des Briefes. Anschließend fährt er mit seiner abwechselnd paraphrasierenden und in Form von Quästionen diskursiv voranschreitenden Auslegung fort. Schon im $\operatorname{Prolog}^{17}$ zu seiner Erklärung fällt es auf, dass er die symbolische Theologie nicht als Wissenschaft im eigentlichen Sinn, sondern als die 'Kunst der Theologie' bzw. die 'Kunstfertigkeit' (symbolicae theologiae ars) qualifiziert; diese Bestimmung wird von ihm im weiteren Verlauf der Kommentierung grundsätzlich beibehalten. ${ }^{18}$ Die Unterscheidung zwischen der Theologie als Wissenschaft (scientia) einerseits, wie sie im Sentenzenkommentar ausgewiesen wurde und nachmalig in der Summa theologiae I in einer umfassenderen Weise geltend gemacht wird, und andererseits der Theologie als Kunst (ars) ist wissenschaftstheoretisch motiviert und begründet. Sie verdient deshalb eine kurze Erläuterung.

In den Frühwerken unterstreicht Albert unter Bezugnahme auf die Stoa (Ps.-Cicero) und Aristoteles (u. a. Anal. Post., De gen. an., Eth. Nic., Metaph.) den praktisch-habituellen, handlungsorientierten Charakter der ars. Sie werde in der Weise wie die scientia durch mehrfache Erfahrung gewonnen. Die Erfahrung aber sei die Erkenntnis des Einzelnen (cognitio singularium), weshalb die ars ein kognitiver, auf konkrete Handlungen bezogener und diese als operatives Prinzip gestaltender Habitus (habitus factivus cum ratione veri) sei. ${ }^{19}$ Häufig wiederholt Albert die Ansicht, dass die ars die Natur nachahmt. Diese auf Aristoteles (Phys. II 2, 194a21-22) zurückgeführte Auffassung verknüpft er mit einer von ihm geprägten, neuplatonisch inspirierten Formel, die Natur sei das Werk (und das Werk-

17 Albertus Magnus, Super Dionysii Epistulas, ep. IX, p.528 v.2-65.

18 Cf. ibid. p.528 v.6-7.11-12.67 - p.529 v.1, p.532 v.12-13.

19 Cf. Albertus Magnus, De natura boni, p.5 v.13-15, p.7 v.3-4; id., De homine, eds. Henryk Anzulewicz/Joachim R. Söder (Alberti Magni Opera Omnia XXVII/2), Münster 2008, p. 143 v.26: "ars est principium et forma illius quod erit" (nach Aristoteles, De generatione animalium II 1, 735a2-3); ibid. p.453 v.62 - p.454 v.10; id., De bono tr.1 q.4 a.7, ed. Heinrich Kühle et al. (Alberti Magni Opera Omnia XXVIII), Münster 1951, p.64 v.53-56; q.5 a.1, p.73 v.86; tr.4 q.1 a.2, p.226 v.6-7. Seine Auffassung bleibt dieselbe auch in den späteren Schriften, cf. id., Super Dionysium De caelesti hierarchia c.3, eds. Paul Simon/Wilhelm Kübel (Alberti Magni Opera Omnia XXXVI/1), Münster 1993, p.49 v.59-60; c.15, p.239 v.28-29 (in den beiden Fällen nach Arist., Eth. Nic. VI 4, 1140a10.20-21); Super Ethica VI lect.6, ed. Wilhelm Kübel (Alberti Magni Opera Omnia XIV/2), Münster 1987, p.429 v.9sqq.; Ethica VI tr.2 c.5, ed. Aug. Borgnet (Alberti Magni Opera Omnia VII), Paris 1891, p.414b-415a. 
zeug) der Intelligenz (opus naturae est opus intelligentiae). Die Analogie von ars und natura erblickt er einerseits in deren Tätigkeit, andererseits in der instrumentellen Funktion der einen wie der anderen gegenüber der wirkenden -sei es göttlichen, sei es menschlichen- Intelligenz. ${ }^{20}$

In seinen beiden Ethikkommentaren erläutert Albert, weshalb Aristoteles (Eth. Nic. VI 3, 1139b15-17) die ars als die erste und elementarste intellektuelle Tugend bestimmte, die durch die Wissenschaft (scientia), die Klugheit (prudentia), die Weisheit (sapientia) und die Vernunfteinsicht (intellectus) überhöht wird. ${ }^{21}$ Diese mit der ars beginnende, aufsteigende Ordnung der dianoetischen Tugenden entspreche, wie er Eustratius folgend die Auffassung des Aristoteles interpretiert, dem naturgemäBen Voranschreiten in der intellektuellen Vollendung. Die Wissenschaft (scientia) als intellektueller Habitus stütze sich auf den Mittelbegriff, der zwar sicher und notwendig, aber dennoch mit Zeit und Raum verknüpft sei. Hinsichtlich der Verständnisgewissheit (certitudo comprehensionis) sei sie sicherer als die Klugheit (prudentia). Die Letztere stehe unter dem humanen und unter dem göttlichen Gesetz, insofern sie von der Intelligenz auf natürliche Weise in die Seele eingeprägt werde und sich eines Mittelbegriffs bediene, der von Raum und Materie völlig getrennt sei. Als vom göttlichen Gesetz reguliert, gelange sie zur Weisheit und werde dadurch vollkommener als die Wissenschaft. Die ars hingegen sei von der Weisheit am weitesten entfernt und als die erste Sprosse der dianoetischen Tugendleiter auf die äußere Aktivität gerichtet. Ihr kognitiver Gehalt und ihre Wertigkeit entsprechen dem sie generierenden, von Raum und Zeit gänzlich abhängigen Mittelbegriff. ${ }^{22}$ Dennoch vermittelt die ars von sich aus (de se), schreibt Albert im späteren Ethikkommentar, die Wahrheit, wenngleich ihre Erzeugnisse (artificiata) von den Kunstregeln bisweilen abweichen ${ }^{23}$. Mit dieser Bestimmung der ars als die basale dianoetische Tugend, welche die Erkenntnis vermittelt und eine äußere

20 Zur Analogie von ars und natura cf. Albertus Magnus, De sacramentis tr.2 q.6, ed. Albert Ohlmeyer (Alberti Magni Opera Omnia XXVI), Münster 1958, p.23 v.21-23; id., De homine, p.27 v.42, p.50 v.17, p.104 v.33, p.134 v.26, p.141 v.48-53. Zur Formel opus naturae est opus intelligentiae cf. Weisheipl (1980); Hödl (1994). Zur Verknüpfung der beiden Gedanken cf. Anzulewicz (2008), 29-35; Takahashi (2008).

21 Albertus Magnus, Super Ethica VI lect.4-11, p.416-473; Ethica VI tr.2, p.407-445.

22 Cf. Albertus Magnus, Super Ethica VI lect.4, p.417 v.49-80, p.418 v.8-19.

23 Albertus Magnus, Ethica VI tr.2 c.1, p.408b: "ars et prudentia de se verum dicunt, quamvis artificiata et prudentialia aliquando transcindant a principiis artis et scientiae". 
Tätigkeit leitet, beenden wir unseren Exkurs über Alberts begriffliche Analysen von ars, eines für unseren Zusammenhang relevanten Aspektes seines komplexen ars-Verständnisses. ${ }^{24}$

\section{III}

Die inhaltlichen und hermeneutischen Koordinaten seines Kommentars zum neunten Brief des Dionysius leitet Albert aus Buch Hosea 12,1025 ab und stellt die zeichenhaft-symbolische Dimension der prophetischen Offenbarung, ihre Expressivität und ihre Wirksamkeit, welche über die Wortsprache hinausgeht, in den Vordergrund. Er weist auf zwei Aspekte hin: (1) auf den Gegenstand der symbolischen Theologie, der in der Vielzahl und Vielfalt der theologischen Figuren und Symbole besteht, und (2) auf die Methode ihrer Interpretation, die er als 'das Hineinsehen in die Ähnlichkeit' (per inspectionem similitudinis) bezeichnet. ${ }^{26}$

Mit Bezug auf die Vielzahl der theologischen Symbole und Visionen unterstreicht Albert, dass Gott ihr Urheber ist und dass sie unserer Auffassungsgabe angemessen sind. Denn einerseits sind Menschen bei der Erkenntnis des Göttlichen naturgemäß an die Gegenstände der Sinneswahrnehmung angewiesen. Andererseits sind die vielen Symbole notwendig wegen der Vielfalt von Gottes Kraft, die in unzähligen Wirkungen widerscheine, aus denen Gott gleichsam induktiv, wie die Hl. Schrift (Ex 33,23; Jes 6,1) lehrt, erkannt werde. Die Aussagen aus dem Alten Testament und die auf Aristoteles zurückgehende Feststellung, dass menschliche Erkenntnis grundsätzlich bei der Sinneswahrnehmung ansetzt, weisen auf die anthropologischen Voraussetzungen der Gotteserkenntnis hin, denen die symbolische Theologie, wie Albert zeigen will, auf eigenartige Weise Rechnung trägt. ${ }^{27}$

24 Die ars-Tradition und den ars-Begriff untersucht mit Bezug auf Alberts Kommentar Super Dionysium De divinis nominibus Weismantel (2008/2009), 25-30. Umfassende begriffsgeschichtlich-lexikographische Orientierung bietet Merle (1986) 95-133.

25 "'Ego visiones multiplicavi eis et in manibus prophetarum assimilatus sum', Os. XII (10)": Albertus Magnus, Super Dionysii Epistulas, ep. IX, p.528 v.2-4. Der als solcher formal nicht gekennzeichnete Prolog umfasst in der kritischen Ausgabe, wie oben festgehalten (Anm.16), die ersten drei Textabschnitte des Kommentars, p.528 v.2-65.

26 Albertus Magnus, ibid. p.528 v.10-15.54-55.

27 Albertus Magnus, Super Dionysii Epistulas, ep. IX, p.528 v.16-24.44-53. Cf. id., De homine, p.1 v.21-22, p.446 v.47-49, p.473 v.14-24. Rodolfi (2006). 
Die Visionen differenziert Albert unter zweierlei Gesichtspunkten jeweils dreifach, nämlich seitens des wahrnehmenden Subjektes und vonseiten des Sehgegenstandes. Dem erstgenannten, durch Augustinus festgelegten Kriterium zufolge gibt es drei Stufen von Visionen. Die erste Stufe sei eine sinnenhafte oder körperliche Vision (visio sensibilis/corporalis), die höhere Form vollziehe sich in der Vorstellung oder im Traum (visio imaginaria/per somnium), die höchste Form indes sei ein Schauen intellektueller Art (visio intellectualis), welches den Höhepunkt kontemplativer Betrachtung in diesem Leben darstelle. Bei der Unterscheidung der Visionen nach Sehgegenständen nennt Albert ebenfalls drei Gruppen, nämlich Erscheinungen in Menschengestalt, in der Gestalt des Feuers und in den sonstigen Formen. ${ }^{28}$

Das 'Hineinsehen in die Ähnlichkeit' als die Methode der symbolischen Theologie adaptiert Albert von Aristoteles (De divinatione per somnum, c.2), der sie als den Weg zur Beurteilung und Interpretation der Träume nennt. Insofern wir in die Eigenschaften der Dinge, die bildhaft Gott umschreiben, hineinschauen, steigen wir, schreibt Albert, 'aufgrund der Ähnlichkeit in der Weise der Proportionalität' (per similitudinem proportionalitatis) zur Erkenntnis des Göttlichen auf. ${ }^{29}$

Aus diesem Überblick über die einleitenden Themen, die Albert im Prolog kurz abhandelt, konnte man ersehen, dass und wie der Doctor universalis die wissenschaftstheoretischen und hermeneutischen Voraussetzungen und Vorgaben des Kommentars zur neunten Epistel des Dionysius mit dem Ziel bestimmt und erläutert, die symbolische Theologie als 'Kunst' (ars) im epistemischen Sinn zu erweisen. Es war nicht zu übersehen, dass er für diesen Zweck viele dem kommentierten Text formal und sachlich fremde Elemente zu einer in sich stimmigen Einheit integrierte. Hierzu gehören die von Aristoteles inspirierte Wissenschafts- und Methodenlehre, die Bibelexegese, die systematische (augustinische) Theologie sowie die erkenntnispsychologischen und hermeneutischen Aspekte der Aristotelischen Naturphilosophie. In letztgenanntem Punkt kam dem Lehrstück des Aristoteles über die prophetischen Träume und die Kunst ihrer Deutung (De insomniis und De divinatione per somnum) eine wichtige

28 Albertus Magnus, Super Dionysii Epistulas, ep. IX, p.528 v.24-39.

29 Ibid. p.528 v.54-58: "Modus autem expositionis est per inspectionem similitudinis, sicut etiam dicit Philosophus de expositionibus somniorum, quando inspectis proprietatibus rerum, sub quarum figura describitur deus, per similitudinem proportionalitatis in divina conscendimus". 
Funktion zu. ${ }^{30}$ Alberts kreativer Umgang mit dem Dionysischen Text ermöglichte ihm, wie sich im Folgenden noch zeigen soll, die Entfaltung der symbolischen Theologie zu einer ars manuductionis in Dei cognitionem, einer theologischen Kunst, die durch die Bildersprache der Offenbarung, ihre Metapher und Symbolik den Menschen und die Kirche auf ihrem Weg durch die Zeit gleichsam an der Hand zur Erkenntnis göttlicher Geheimnisse hinführt. ${ }^{31}$

IV

Die Darstellung der allgemeinen 'Kunst' der symbolischen Theologie bildet das Hauptstück von Alberts Kommentar zur neunten Epistel des Dionysius. Im darauf folgenden, letzten Teil wird der theologische Sinngehalt jener drei Symbole entfaltet, die Dionysius im Brief an Titus erläutert. ${ }^{32}$ Dass hierbei 'Nahrung', 'Mischgefäß' und 'Haus der Weisheit' (alimentum, crater, domus sapientiae) als theologische Symbole gewählt wurden, war kein Zufall, sondern hatte seinen Grund in der Bibel, wie Albert mit dem Hinweis auf Spr 9 vergegenwärtigt, wo sie als Parabel des Salomo erscheinen..$^{33}$ Alle Fragen, die Albert in den beiden Teilen des Kommentars thematisiert, haben wir aus dem Text extrahiert und in einem analytischen Index im Anhang erfasst. Nur einige von ihnen, die offensichtlich von übergeordneter Bedeutung sind, können hier kurz besprochen werden. Wir beschränken uns in Bezug auf das erste Hauptstück auf Alberts Standpunkt zu den Fragen nach der Notwendigkeit der symbolischen Theologie (IV.1), nach den Symbolen und ihrer Bedeutung (IV.2) sowie der allgemeinen Methode ihrer Auslegung (IV.3). Zum Schluss folgen eine Anmerkung zum Verhältnis von symbolischer und mystischer Theologie (V) und ein quellenkritischer Exkurs über den hermeneutischen Ertrag

30 Zur Typologie der Visionen cf. Anzulewicz (1999), 278-284; Schlosser (2000), 50103. Die beiden Opuscula des Aristoteles (De insomniis und De divinatione per somnum) hatte Albert erstmalig vor der Kommentierung des Corpus Dionysiacum in seinem Werk De homine diskutiert und später erneut einer Kommentierung unterzogen; cf. Albertus Magnus, De homine, p.381 v.18 - p.392 v.68; id., De somno et vigilia 1.3, ed. Aug. Borgnet (Alberti Magni Opera Omnia IX), Paris 1890, 177-207.

31 Cf. Albertus Magnus, Super Dionysii Epistulas, ep. IX, p. 528 v.52-53, p.529 v.60-62.79-81.

32 Für die Angaben zur zweiteiligen Hauptstruktur des Textes siehe ibid. p.528 v.66 - p.529 v.3; 1 . Hauptstück: p.529 v.3 - p.542 v.23; 2. Hauptteil: p.542 v.24 - p.546 v.12.

33 Cf. Albertus Magnus, Super Dionysii Epistulas, ep. IX, p.542 v.28. 
aus der Indienstnahme des philosophischen und naturkundlichen Wissens in der symbolischen Theologie (V).

1. Die Notwendigkeit der symbolischen Theologie steht für Albert außer Zweifel. Er sieht sie durch die Anwendung der Parabel als Methode in der Hl. Schrift legitimiert und begründet sie in Anlehnung an die Schriften De Mystica theologia und De caelesti hierarchia des Dionysius wie folgt: Da wir über Gott mit größerer Gewissheit wissen, was er nicht ist als was er wirklich ist (wie aus der Mystischen Theologie hervorgeht), eigne sich das, was von ihm offenkundiger fern sei, am ehesten, uns zu ihm zurückzuführen. Deshalb sei die Verwendung der Sinnbilder, Gleichnisse und Metaphern, kurzum Symbole, als Methode für die Theologie am meisten angemessen. ${ }^{34}$ Die Theologie überragt alle Wissenschaften, über die der Mensch verfügt, auch die erhabenste von ihnen, die Metaphysik. ${ }^{35}$ Denn sie alle entsprechen seiner natürlichen, im einfachen Intellekt und in der diskursiven Vernunft gründenden Erkenntnisfähigkeit. ${ }^{36}$ Weil die theologische Wissenschaft über dieses Entsprechungsverhältnis hinausgeht, ist es notwendig, dass eine Entsprechung auf natürliche Weise hergestellt wird. Die symbolische Theologie leistet die Vermittlung, indem sie uns, wie Albert in der Sprache des Dionysius zum Ausdruck bringt, gleichsam an der Hand in den innersten Teil der höchsten Wissenschaft, welcher von Gott handelt, führt. ${ }^{37}$

34 Albertus Magnus, Super Dionysii Epistulas, ep. IX, p.529 v.12sqq., v.58-63: "de deo verius scimus, quid non est quam quid est, sicut patuit ex Mystica theologia, et ideo ea quae sunt manifestioris remotionis ab ipso, sunt magis convenientia ad reducendum nos in deum; et ideo modus per symbola est maxime conveniens theologiae". Albert stellt eine Verbindung zwischen der mystischen Theologie des Dionysius, die er zuvor einer Kommentierung unterzog, und der symbolischen Theologie über die negativen Prädikationen über Gott her, wodurch er einen weiterführenden Weg zur Erkenntnis der Andersheit Gottes gewinnt. Cf. Meis (2006), 541-574.

35 Albertus Magnus, Super Dionysii Epistulas, ep. IX, p.529 v.19-20.29.35.68-69. Alberts wissenschaftssystematisches Konzept umfasst fünf Hauptfächer -Logik, Ethik, Naturphilosophie, Mathematik und Metaphysik-, die er unter ein dreiteiliges Gefüge subsumiert, nämlich in philosophia rationalis (Logik), philosophia moralis (Ethik einschließlich der Politik und Ökonomik) und philosophia realis (Naturphilosophie, Mathematik und Metaphysik); cf. Albertus Magnus, De IV coaequaevis tr.4 q.61 a.4, p.656a.

36 Albertus Magnus, De IV coaequaevis 1.c.

37 Albertus Magnus, Super Dionysii Epistulas, ep. IX, p.529 v.65-70: "scientiae altissimae proportionatae nobis, cuiusmodi est metaphysica, modus debet esse altissimus per 
Die Vermittlung der Offenbarungswahrheit durch Metaphern und Gleichnisse, die sich an partikuläre Sachverhalte und Eigenschaften der Dinge anlehnen, wie es in der Hl. Schrift geschieht, billigt weder eine einfältige Vulgarisierung der Theologie noch stellt sie ihre Wissenschaftsfähigkeit und ihren Status als Wissenschaft in Frage. Als eine der Frömmigkeit gemäße, praktisch ausgerichtete Wissenschaft ist sie, wie Albert in Summa theologiae I unter Hinweis auf Röm 1,14 (Sapientibus et insipientibus debitor sum) erklärt, sowohl für die Weisen wie auch für die Ungebildeten heilsnotwendig. ${ }^{38}$ Es war also erforderlich, dass sie solche Methoden anwendet, durch die sie alle Menschen anspricht. Der Modus einer Wissenschaft und einer Kunstfertigkeit hingegen sei nicht für alle Adressaten der Heilsbotschaft, die alle Bereiche der menschlichen Existenz berührt, angemessen. Bildersprache und konkrete Ausdrucksweise seien selbst für Weise und Gebildete alles andere als trivial, weil den geschilderten, partikulären Sachverhalten allgemeine theologische Wahrheiten innewohnen. Die Väter hätten viele solcher universalen Heilswahrheiten aus konkreten Beispielen und Bildern abstrahiert, erschlossen und in ihren Schriften in wissenschaftlicher Form überliefert. Wenn also die bildhaft und konkret dargestellten theologischen Wahrheiten der Ungebildete und der Gelehrte jeweils anders begreifen, verstehen sie beide diese dennoch, wie Albert im Anschluss an Hieronymus (Epist. 53) hervorhebt, im Sinne einer erbauenden Unterweisung.

Die Verwendung von Metaphern und Symbolen ist in der Theologie, anders als in der Philosophie und allen weltlichen Wissenschaftsdisziplinen, die dem Aristotelischen Wissenschaftsideal verpflichtet sind, wie gesagt, legitim, erforderlich und notwendig. Aufgrund seiner Bindung an Raum und Zeit verhält sich der menschliche Intellekt dem an sich Offenbarsten gegenüber, dem göttlichen Licht nämlich, wie das Auge der Fledermaus, das nur mit Dunkelheit versetztes Licht aufnimmt. Deshalb formt der Intellekt das Einfachste und Offenbarste zum Vorstellbaren und Sinnenhaften, um dadurch, gleichsam an der Hand geführt, zu dem Unsichtbaren und Einfachsten emporsteigen zu können und von diesen

prima principia; sed theologia est altior, excedens nostram proportionem, et ideo oportet, quod manuducamur in ipsam per connaturalia nobis”. Cf. id., Super Dionysium De caelesti bierarchia c.1, p.13 v.8-42; Summa theologiae I, tr.1 q.5 c.1, ed. Dionysius Siedler et al. (Alberti Magni Opera Omnia XXXIV/1), Münster 1978, p.16 v.72, p.17 v.25.

38 Hierzu und zum Folgenden: Albertus Magnus, Summa theologiae I tr.1 q.5 c.1, p.16 v.37-65. 
auf eine ihm mögliche Weise Kenntnis zu empfangen. Die Auffassung von den Möglichkeiten und Modi der natürlichen Erkenntnis des Göttlichen, welche dem menschlichen Intellekt eigentümlich sind, begründet Albert philosophisch und theologisch, indem er hierbei einerseits an die einschlägigen Topoi bei Aristoteles (Metaph. II 1) und Boethius (De cons. philos. IV pr.6) anknüpft, anderseits sich an Augustinus (De Gen. ad litt. XII), Gregor den Großen (Hom. 11 in Ev.) und vor allem Dionysius (De cael. bier. c.1) anlehnt. ${ }^{39}$

Die symbolische Theologie verwendet auch poetische Mittel. Sie tut dies in einer Weise, die nicht vergleichbar mit jener in der Dichtkunst ist, mag diese in den Dienst der Philosophie genommen werden. Die Dichtung sei Selbstzweck; sie täusche absichtlich durch fiktive, dem Vernunfturteil zuvorkommende Vorstellungen, die Vergnügen oder Spannung bewirken sollen, um den Geist an sie zu fesseln, und zwar dadurch, wofür diese Kunst entweder eintritt oder wogegen sie ist. Wenn sie auf etwas hinausführt, dann nur auf den Menschen als ihren Adressaten, auf den sie zugemessen und ihm gänzlich verhältnismäßig sei. ${ }^{40}$ Ihre staunenswerten Inhalte seien ein Produkt des Menschen und in allem, was sie vergegenwärtigt und worauf sie hinauslenkt, eine Fiktion. Sie sei deshalb verführerisch und falsch, wie Albert in der Summa theologiae I und anderswo schreibt. ${ }^{41}$ Die Theologie indes bedient sich der Symbolik nicht in der Absicht, an die Bilder zu fesseln, sondern um von ihnen zu lösen und durch die bildlich vermittelten Ähnlichkeiten auf das hin

39 Ibid. p.16 v.67sqq.; cf. id., Super Matthaeum 13,1, ed. Bernhard Schmidt (Alberti Magni Opera Omnia XXI/1), Münster 1987, p.394 v.5-8.

40 Albertus Magnus, Super Dionysii Epistulas, ep. IX, p.529 v.71-77: “modus symbolicae theologiae est alius a modo poetriae, quia modus poetriae est falsus; est enim intentio poetae, ut inhaereat anima suis figmentis, in quibus concipiat vel horrorem vel delectationem eius quod intendit persuadere vel dissuadere, et si per illas fictiones in aliquid ducatur, illud totum est proportionatum nobis; sed theologia non proponit nobis symbola, ut eis adhaereatur, sed intendit removere ab eis et per similitudines in eis repertas manuducere in id quod est supra nostram proportionem." Cf. ibid. p.529 v.85 - p.530 v.3: "poeta autem proponit ea quae deficiunt a ratione, quibus ratio non consentiret, et ideo praevenit (iudicium rationis) quibusdam miris stuporem excitantibus, quo anima detinetur".

41 Albertus Magnus, Summa theologiae I tr.1 q.5 c.2, p.18 v.17-23: "In poesi autem philosophorum mira, ex quibus fabula componitur, ex fictione humana oriuntur et propter repraesentationem ad humana dirigunt, et ideo deceptoria sunt et mendosa". Cf. id., Super Dionysii Epistulas, ep. VII, p.504 v.48-59. Simon (1978), 96-99. 
zu führen, was unser Fassungsvermögen übersteigt. ${ }^{42}$ Die Dichtung der Hl. Schrift und des Theologen gehe auf die göttliche Weisheit als ihren Urheber zurück und lenke den Menschen auf die sicherste Weise durch Ähnlichkeiten, die seiner Auffassungsgabe entsprechen, auf das Undarstellbare und Immaterielle, auf das hin, wodurch sie selbst - die göttliche Poesie- hervorgebracht wurde. Sie komme der Vernunft nicht zuvor, sondern sie zeige ihr mit angemessenen Mitteln das auf, was sie übersteige. Ihre Gewissheit wird durch die Gewissheit ihres Ursprungs und ihrer Zielbestimmung verbürgt. ${ }^{43}$

Die bisher referierte Begründung der Notwendigkeit der symbolischen Theologie entfaltete Albert, wie eingangs festgehalten, selbständig in Form einer Quästion, die er nach dem Prolog und einer kurzen Strukturierung des Dionysius-Textes mit Blick auf seine Auslegung der eigentlichen Paraphrase vorausschickte. Zu Beginn der Paraphrase kam er auf die von Dionysius genannten zwei Gründe für die Notwendigkeit der ars der symbolischen Theologie zu sprechen. Der erste Notwendigkeitsgrund liegt, wie er dem kommentierten Text entnimmt, in den Symbolen, die von Menschen, deren Glaube unvollkommen ist, als unangemessen zur Darstellung der göttlichen Wahrheit betrachtet werden. Die Geheimnisse Gottes empfange man nicht, meinen sie, durch 'Unreines' (immunda) - diesen Dionysischen Ausdruck deutet Albert mit 'Argumentation'-, sondern in der Frömmigkeit durch erhabenere Ähnlichkeiten. Die vermeintliche Inkongruenz zwischen der Rede von Gott und der hierfür gebrauchten, mitunter pejorativ anklingenden Symbolik sowie der Verlust des Glaubens bei Menschen, die von den Sinnesbildern gefesselt würden und ihren verborgenen Wahrheitsgehalt nicht erkennen könnten, diese Schwierigkeiten machen eine Erklärung der Kunst der symbolischen Redeweise über Gott notwendig. ${ }^{44}$

42 Albertus Magnus, Super Dionysii Epistulas, ep. IX, p.529 v.78-81: "theologia non proponit nobis symbola, ut eis adhaereatur, sed intendit removere ab eis et per similitudines in eis repertas manuducere in id quod est supra nostram proportionem".

43 Albertus Magnus, Summa theologiae I tr.1 q.5 c.2, p.18 v.11-17: "sacra scriptura poeticis utitur ex divina sapientia formatis et figuratis, in quorum figuris secundum proportionem similitudinum resultant infigurabilia et immaterialia, eo quod ab illis et ad illa formata et figurata sunt, et ideo certissima sunt, ex certissimis oriuntur et ad certissima dirigunt”. Id., Super Dionysii Epistulas, ep. IX, p.529 v.83-85: "theologus non intendit praevenire iudicium rationis, sed rationi manifestat ea quae sunt supra proportionem ipsius, per connaturalia sibi”.

44 Albertus Magnus, Super Dionysii Epistulas, ep. IX, p.530 v.43-66. 
Der andere Notwendigkeitsgrund für diese ars leitet sich, wie Albert dem Text seiner Vorlage entnimmt, aus der zu vermittelnden Glaubenswahrheit ab. Es sei notwendig, dass sie durch die Auslegung von den Symbolen gelöst und in ihrer Reinheit wiedergegeben werde. Denn es ist nicht offenkundig, was es bedeutet, wenn in der Hl. Schrift Gott als 'Quelle des Lebens' (fons vitae: Ps 35,1) oder 'Quelle der Weisheit' (fons sapientiae: Sir 1,5) bezeichnet wird. Diesbezügliche Erläuterungen des Dionysius sind bisweilen von höchster philosophischer Dichte und erfordern weitere Auslegung, die Albert sowohl in der Paraphrase als auch in den eingewobenen Quästionen seines Kommentars geistvoll durchführt. Ein Beispiel dafür, das sich uns bereits im Zusammenhang mit der Dionysischen Auslegung der Gottesbenennung 'Quelle' bietet, sind seine Interpretationen von virtus simplex als dem Synonym der göttlichen Wahrheit und der cognitio omnium cognitionum als dem Inbegriff göttlicher Erkenntnis und dem eigentlichen Prinzip jeder Sache sowie aller Erkenntnis. ${ }^{45}$ Hierbei zeigt sich, dass und wie kognitiv leistungsfähig und theologisch fruchtbar die neuplatonische philosophische Tradition ist, welche die Schriften des Dionysius prägt, wenn Albert z. B. die innergöttlichen Hervorgänge oder die Erkenntnis als solche emanatistisch deutet. ${ }^{46}$

Die Notwendigkeit der Loslösung von den Symbolen mündet nicht in Aporie, obgleich Dionysius in De caelesti hierarchia darauf hinzudeuten scheint, wenn Albert ihn das Wort reden lässt: "EE]s ist unmöglich, dass der göttliche Strahl uns auf andere Weise schimmert, wenn nicht durch

45 Ibid. p.530 v.79 - p.531 v.64.

46 Ibid. p.531 v.34-40. 55-64: "dicitur fons effusus ad se, quia non habet aliud principium suae effusionis, sed a se sibi est vel ad se, quia in simile sibi secundum formam effunditur, quia aqua est eiusdem rationis in fluvio et fonte; vel potest referri ad emanationem personarum in divinis, in quibus pater fundit filium non extra suam substantiam. (...) dicitur una cognitio omnium cognitionum divina cognitio quantum ad se, inquantum ipse cognoscendo se cognoscit omnia alia, quia est principium proprium uniuscuiusque rei vel etiam omnium cognitionum, quibus alia cognoscunt, et dicitur una cognitio omnium cognitionum sicut principium efficiens et forma universalis exemplaris, a qua fluit omnis cognitio, quia quae sunt in multis, oportet inveniri in uno primo, a quo in omnia alia fluunt." Cf. Albertus Magnus, Metaphysica 1.1 tr.1 c.4, ed. Bernhard Geyer (Alberti Magni Opera Omnia XVI/1), Münster 1960, p.6 v.26-42,71-72; id., De causis et processu universitatis a prima causa 1.1 tr.4 c.1, ed. Winfried Fauser (Alberti Magni Opera Omnia XVII/2), Münster 1993 p.42 v.35sqq., bes. v.4563; c.4, p.46 v.69sqq. 
Albertus Magnus über die ars de symbolica theologia des Dionysius Areopagita | 323

verschiedene Arten heiliger Gewänder umhüllt. ${ }^{647}$ Die sich anscheinend ausschließenden Sichtweisen auf die Möglichkeiten einer unmittelbaren Gotteserkenntnis vermittelt Albert miteinander, indem er sie als Anfang und Endziel der Kontemplation des Menschen auf dem Weg zu Gott interpretiert: ,[H]insichtlich des Anfangs der Kontemplation ist es notwendig, dass uns auf irdischem Lebensweg der Strahl der göttlichen Wahrheit durch Symbole leuchtet, aber am Ende kommen wir durch die Symbole zu der nackten Wahrheit selbst“، 48

2. Sinn und Bedeutung der Symbole in der Theologie bestehen nach Albert in der Manifestation der geistigen Wirklichkeit durch Sinnenhaftes aufgrund ihrer Ähnlichkeit nach Art der Proportionalität (per similitudinem proportionalitatis). Die Symbole als solche werden durch das 'Hineinsehen in diese Ähnlichkeit' (inspectio similitudinis) erkannt und interpretiert. Wie dies geschieht, erläuterte er u. a in folgender Weise: ${ }^{49}$

" $[\mathrm{M}]$ an muss sagen, dass obwohl derartige Symbole, insofern sie gewisse Sinnesgegenstände sind, die Wahrheit verdecken, dennoch, insofern

47 Albertus Magnus, Super Dionysii Epistulas, ep. IX, p.530 v.72sqq., hier p.531 v.9-11: "sicut dicitur in primo capitulo Caelestis hierarchiae, 'impossibile est nobis aliter divinum radium splendere nisi varietate sacrorum velaminum circumvelatum".

48 Albertus Magnus, Super Dionysii Epistulas, ep. IX, p.531 v.29-33: “quantum ad principium contemplationis oportet, quod in statu viae per symbola luceat nobis radius divinae veritatis; sed in fine per symbola in ipsam nudam veritatem venimus".

49 Albertus Magnus, Super Dionysii Epistulas, ep. IX, p.530 v.4-28: "dicendum, quod quamvis huiusmodi symbola occultent veritatem, inquantum sunt sensibilia quaedam, tamen inquantum sunt relata ad spiritualia, manifestant ea. Referuntur autem symbola sensibilium in spiritualia per similitudinis inspectionem, ut dictum est, non quae sit secundum participationem eiusdem qualitatis, quia omnino differentes sunt qualitates corporalium et spiritualium, neque per proportionem ad illa, quia improportionabilia sunt, nec est eis quantitas eiusdem rationis, sed per similitudinem proportionalitatis, in qua oportet quattuor facere, scilicet accipere proprietatem rei sensibilis et comparare eam ad suum actum et similem comparationem invenire proprietatis rei spiritualis ad suum actum, sicut proprietas leonis est animositas, per quam vincit omnia animalia sibi resistentia; similiter deus sua virtute superbos et resistentes sibi deicit. Non est igitur neque virtus neque actus victoriae eiusdem rationis in leone et deo, sed tantum comparatio, quia sicut se habet virtus leonis ad suam victoriam, ita virtus divina ad suam. Et haec est similitudo proportionalitatis, quae semper est ad minus inter quattuor vel re vel ratione, quando uno utitur ut duobus, sicut si dicatur: sicut se habet quattuor ad duo, ita sex ad tria, vel: sicut sex ad quattuor, ita novem ad sex." Cf. ibid. p.533 v.48sqq. 
sie auf das Geistige bezogen sind, sie [die Wahrheit] aufzeigen. Die Symbole des Sinnenhaften führt man aber, wie gesagt, auf das Geistige durch das Hineinsehen in die Ähnlichkeit zurück, [allerdings] nicht eine solche [Ähnlichkeit], die auf der Teilhabe an ein und derselben Eigenschaft beruht, weil die Eigenschaften des Körperlichen und des Geistigen gänzlich verschieden sind, und auch nicht eine [Ähnlichkeit] aufgrund der Verhältnismäßigkeit zu den beiden, weil sie unverhältnismäßig zueinander sind und ihnen [-dem Körperlichen und dem Geistigen-] auch keine Größe von ein und derselben Art und Weise eignet, sondern [man führt die Symbole des Sinnenhaften auf das Geistige] aufgrund der Ähnlichkeit nach Art der Proportionalität. Hierbei muss man viererlei Dinge tun, nämlich (1) eine bestimmte Eigenschaft eines Sinnesgegenstandes nehmen und (2) sie ihrer Wirkung gegenüberstellen, dann (3) ein ähnliches Verhältnis der Eigenschaft eines geistigen Gegenstandes zu (4) seiner Wirkung finden, z. B.: die Eigenschaft des Löwen ist der Mut, durch welchen er alle Tiere, die ihm Widerstand leisten, besiegt; ähnlich wirft Gott durch seine Kraft die Hochmutigen und die sich ihm widersetzen nieder. Weder die Kraft also noch der Siegesakt ist bei Löwen und bei Gott von ein und derselben Art und Weise, sondern nur eine Verhältnisgleichheit, weil so wie sich die Kraft des Löwen zu seinem Sieg verhält, so sich die göttliche Kraft zu ihrem [Sieg]. Und diese ist die Ähnlichkeit nach Art der Proportion, die immer zwischen mindestens vier [Größen] der Sache oder dem Begriff nach besteht, wenn man sich des einen wie zweier bedient, so als wenn man sagte: in der Weise wie sich vier zu zwei verhält, so sich sechs zu drei, oder: so wie sechs zu vier, so neun zu sechs [verhält]."

Bei der Darstellung der allgemeinen Methode der symbolischen Theologie stellt Albert fest, dass Dionysius die Bedeutung der Symbole und die Symbole zum Ausgangspunkt dieser ars macht. Erst im nächsten Schritt erklärt der Areopagite die Methode der Auslegung der Symbole. Diese Vorgehensweise ist nach Albert erklärungsbedürftig, da sie offenkundig in umgekehrter Reihenfolge verlaufen müsste. Die Frage löst er mit der Unterscheidung zwischen dem Anwendungsmodus und dem Modus der didaktischen Vermittlung der ars de symbolica theologia. Im Modus der Anwendung (in usu artis), der die natürliche Ordnung (ordo naturae) der symbolischen Theologie verkörpert, setzt sie mit der Auslegung, so Albert, beim inneren Gehalt und der Bedeutung der Symbole an. Ihr Ziel ist die Erkenntnis der symbolisch dargestellten Ähnlichkeit mit den natürlichen Eigenschaften, welche sich aus der äußeren, sinnenhaften Gestalt der Symbole erschließt. Bei der didaktischen Vermittlung dieser ars hin- 
Albertus Magnus über die ars de symbolica theologia des Dionysius Areopagita | 325

gegen (in tradendo artem), wird der Lehrordnung (ordo doctrinae) Rechnung getragen und deshalb von den Symbolen ausgegangen. Die Kenntnis der natürlichen Eigenschaften einer Sache ist nämlich die Voraussetzung für die Zuweisung einer Symbolfunktion an diese Sache, deren Sinngehalt anschließend befragt wird. ${ }^{50}$

3. Die Erklärung der Methodenfrage, die wie ein roter Faden die Epistel des Dionysius durchzieht, setzt Albert mit Blick auf die Bedeutung und Funktion von zwei Arten der Symbole, die er bei Dionysius zu erkennen vermag, fort. Er unterscheidet zwischen einer vollkommenen und einer defektiven Art der symbolischen Bezeichnung bestimmter theologischer Sachverhalte. Die erstgenannte, ideale Art der Bezeichnung wird der Repräsentation einer offenbar weitestgehend vollkommenen Wirklichkeit zugeordnet, wie der Hervorgang der Schöpfung, ihre natürlichen Eigenschaften und Kräfte, innere und äußere Geordnetheit sowie ihre Rückwendung auf Gott als ihr Ziel. In der anderen Art der symbolischen Darstellung kommt zwar ein durch Sünde bedingter Mangel zur Geltung, aber diese Symbole, mögen sie auch nur fromme Dichtung sein, sollen dennoch den Menschen zur göttlichen Wahrheit hinführen. ${ }^{51}$

Wie aus dem bisher Dargelegten erkennbar sein dürfte, hat sich Albert nicht auf die paraphrasierende Auslegung des Dionysius-Textes beschränkt, sondern er ließ sich von ihm vielfältig, jedoch stets mit Blick auf die Erarbeitung und systematische Darstellung der symbolischen Theologie, die er in der neunten Epistel des Areopagiten entdeckt hatte, inspirieren. Außer den eingangs vorgestellten, wissenschaftstheoretischen Aspekten dieser theologischen Kunst behandelte er eine Reihe von Sachfragen, die vorrangig für die symbolische und mystische Theologie

50 Albertus Magnus, Super Dionysii Epistulas, ep. IX, p.532 v.12-19: “in tradendo artem symbolorum oportet prius ponere propria, quae significantur, quam ipsam symbolorum expositionem, quia in expositione symbolorum attenditur similitudo ad propria, et ideo ignoratis propriis non possent eis adaptari symbolica; sed in usu artis est e contrario, sicut procedit obiectio, quia per symbola ducimur in propria”. Ibid. p.534 v.20-25: "licet secundum ordinem doctrinae symbola sint priora significatis, quia ex illis devenimus in ista, tamen secundum ordinem naturae significata veritas prior est symbolis, quia non componuntur symbola nisi per inspectionem ad veritatem rei, ut per similia figuretur".

51 Albertus Magnus, Super Dionysii Epistulas, ep. IX, p.532 v.20sqq., v.56sqq., p.533 v.6sqq., v.31sqq. 
wichtig sind. Mehrere von ihnen berühren auch das Gesamt der Theologie, nicht nur ihr in mancher Hinsicht anderswo kaum erläutertes Wissenschaftsverständnis, wie z. B. die verschiedenen Unterscheidungen und Bestimmungen des theologicum negotium; die Art und Weise der Beteiligung und der Anteil am 'theologischen Geschäft' der Philosophie sowie ihre Klassifikation; die Frage, wie und mit welchem Ziel der Theologe auf die Gegenstände der Philosophie reflektiert, ${ }^{52}$ ferner der mystische Charakter der symbolischen Theologie $e^{53}$ und ihre den Menschen existenziell und kognitiv vollendende Wirksamkeit, ${ }^{54}$ schließlich der Unterschied in der Interpretation identischer Symbole, die verschiedene Wirklichkeiten repräsentieren. ${ }^{55}$ Alle diese Themen verdienen eingehende Untersuchung, die im Rahmen dieses Beitrags nicht mehr aufgerollt werden kann.

Bevor dieser erste Annäherungsversuch an die ars de symbolica theologia, die Albertus Magnus auf der Grundlage der neunten Epistel des Dionysius Areopagita rekonstruierte und entfaltete, mit einem quellenkritischen Exkurs beendet wird, sei auf das Verständnis von Identität und Differenz, die der Doctor universalis in Bezug auf die symbolische und mystische Theologie im Kommentar des Dionysius-Briefes und in der Summa theologiae I feststellte, eingegangen. Ein Vergleich mit seiner diesbezüglichen Auffassung, die er im Kommentar zu De mystica theologia des Dionysius darlegte und die neuerlich von Prof. Anneliese Meis ${ }^{56}$ eingehend untersucht wurde, muss an dieser Stelle aus dem zuvor erwähnten Grund entfallen.

Symbole, die in der Theologie angewendet werden und Gegenstände der symbolischen Theologie sind, seien mystisch und gottförmig (mystica et deiformia), führt Albert übereinstimmend mit dem Text seiner Dionysischen Vorlage aus, weil sie in sich göttliche Eigenschaften durch gewisse Ähnlichkeiten verbergen. Sie seien mit göttlichem Licht, anders gesagt: mit göttlicher Wahrheit, ausgefüllt. ${ }^{57}$ Folglich vermitteln die Symbole

52 Cf. ibid. p.535 v.10sqq., p.539 v.41sqq.

53 Ibid. p.535 v.66-72. Cf. Albertus Magnus, Summa theologiae I, tr.3 q.13 c.1, p.40 v.54 - p.41 v20.

54 Albertus Magnus, Super Dionysii Epistulas, ep. IX, p.536 v.15-49, p.537 v.1sqq., v.84 - p.538 v.5.10sqq.

55 Ibid. p.541 v.66sqq.

56 Meis (2006), 541-574; ead. (2008), 15-36.

57 Albertus Magnus, Super Dionysii Epistulas, ep. IX, p.533 v.64-70. 
den wahrheitsliebenden Menschen in einer ihnen zugänglichen Weise die verborgene göttliche Wissenschaft. Sie verbergen aber diese vor solchen Menschen, die über die Sinneserkenntnis nicht hinausgehen und das Göttliche verspotten, weil sie es nicht vernehmen. ${ }^{58}$ Wie sich die symbolische Theologie, die Albert als die affirmative Theologie begreift, zur mystischen Theologie, die als negative Theologie gilt, verhält und in welcher Hinsicht die symbolische Theologie mit der mystischen Theologie nominell gleichgesetzt wird, erklärt er wie folgt: ${ }^{59}$

“die göttlichen Negationen heißen 'mystisch' im materiellen Sinn, weil indem sie uns von dem entfernen, was uns offenkundig ist, uns in Geheimem belassen, das über unseren Verstand hinausgeht (...); das 'Symbolische' heißt aber das 'Mystische' hinsichtlich des Modus, weil man das Geistige nicht durch den Wortsinn, sondern durch gewisse Figuren vermittelt".

Die symbolische Theologie sei 'geheim und mystisch', schreibt Albert in seiner Dionysius-Paraphrase, hinsichtlich dessen, was durch die Symbole verborgen wird. Sie verfüge in den Symbolen über die bezeichnende Kraft, die über die Sinneswahrnehmung zur Erkenntnis des Geistigen führt, das den Menschen in der Wahrheit vervollkommnet. Die symbolische Theologie ist auch in dem Sinn mystisch, weil sie aus sinnenhaften Zeichen besteht, die sie nicht zu vermitteln hat, und aus dem in ihnen verborgenen Gehalt, den sie vermitteln soll. Durch ihre Vollzüge, den Aufstieg von der Wahrnehmung der Eigenschaften der Sinnesgegenstände zur Erkenntnis des in ihnen verborgenen Geistigen, versetze sie den Menschen in Gott (collocat in deo), wie es Dionysius formulierte, d. h. in das 'Hineinschauen in die göttliche Wahrheit', wie Albert die dionysischen Worte interpretiert. ${ }^{60} \mathrm{Da}$ der Mensch aufgrund seiner Sinnes- und Vernunftbegabung zu diesem Aufstieg, zur Aufnahme einfacher und innerster Wesenheiten der Dinge (simplices quiditates rerum) befähigt ist, gelangt er von der Wahrnehmung äußerer Zeichen zur Anschauung des gottför-

58 Ibid. p.534 v.4sqq.

59 Ibid. p.535 v.66-72: "negationes divinae dicuntur mysticae materialiter, quia removentes nos a manifestis nobis relinquunt nos in quodam occulto quod est supra rationem (...); sed symbolica dicuntur mystica quantum ad modum, quia traduntur spiritualia non per sensum, quem verba faciunt, sed in quibusdam figuris." Cf. ibid. v.19-28.

60 Ibid. p.536 v.18-20.44-49. 
migen Innersten, zu jenen Hervorgängen, welche die Abbilder göttlicher Attribute seien, und werde dadurch vervollkommnet. ${ }^{61}$

Die Erkenntnis durch die Sinneswahrnehmung ist für den Menschen, wie Albert wiederholt zugibt, in diesem Leben natürlicher als die unmittelbare Anschauung der einfachen Wesenheiten der Dinge durch den Intellekt. Deshalb führen wir das Göttliche, sofern es unmittelbar, ohne jede Vermittlung sinnenhafter Symbole angeschaut wird, auf das uns gewohnte und natürliche Sinnenhafte zurück, damit man besser in das Göttliche hineinschauen kann. ${ }^{62}$ Die Theologie in ihrem innersten Teil wird somit offensichtlich zugleich eine symbolische und mystische Theologie. Einer genauen Bestimmung und distinkten Unterscheidung zwischen den verschiedenen Arten des negotium theologicum steht das, wie gesehen, nicht im Weg. Dieser Unterscheidung liegt, wie Albert u. a. in der Summa theologiae I darlegt, das modale Kriterium zugrunde. ${ }^{63}$ Sowohl bei der symbolischen Theologie, die im Kommentar zur neunten Epistel des Dionysius als ars charakterisiert wird, als auch bei der mystischen Theologie handelt es sich um einen Modus der einen theologischen Wissenschaft. Worin dieser Unterschied aber besteht, teilt uns Albert in Anknüpfung an die Dionysische Tradition in seiner Summa theologiae I in folgender Weise mit: ${ }^{64}$

"Wir erkennen Gott nur auf symbolische oder mystische Weise. Auf symbolische Weise erkennen wir Gottes Eigenschaften durch die Proportion einer körperlichen Eigenschaft. Weil die göttliche Eigenschaft

61 Ibid. p.537 v.31-37.50-57.68-93; cf. p.539 v.3-13.

62 Ibid. p.539 v.3-18.

${ }^{63}$ Cf. Albertus Magnus, Summa theologiae I tr.3 q.13 c.1, p.40 v.54 - p.41 v.20.

${ }^{64}$ Ibid. p.40 v.56-78: "Symbolico cognoscimus proprietatem dei proportione proprietatis corporeae. Et cum in infinitum emineat proprietas divina proprietatibus creatorum, procedendo symbolice stamus in infinito et diffundimur in illo, nullum terminum comprehendentes. Mysticis autem procedimus in deum per eminentiam. Mystica enim sunt, quae per se et primo deo conveniunt, per aliud autem et secundario creaturis, ut essentia, vita et intellectus, quae nobis non innotescunt, nisi secundum quod sunt in creatis genere vel specie vel numero, et secundum hoc esse non conveniunt deo, sed eminenter et excellenter in infinitum. Sicut si dicam: 'deus est essentia' et statim illud potentius nego dicens: 'deus non est essentia', eo quod non est essentia, prout nobis essentis innotuit genere vel specie vel numero; et postea infero: 'est essentia super omnem essentiam' nec dicere possum, quantum est super omnem essentiam, eo quod intellectus resolutorius non potest pervenire in aliquod primum, quod non sit aliquid de essentia resolutorum et immixtum eis; deus autem nihil creatorum est, sed eminet omnibus excellenter in infinitum, et ideo iterum intellectus sic procedens stat in infinito et diffunditur in illo." 
Albertus Magnus über die ars de symbolica theologia des Dionysius Areopagita | 329

die Eigenschaften der Geschöpfe ins Unendliche überragt, kommen wir auf symbolische Weise voranschreitend im Unendlichen zu stehen und wir verströmen uns in ihm, indem wir an keine Grenze gelangen. Auf mystische Weise nähern wir uns Gott durch das Hervorragende. Das Mystische ist nämlich das, was an sich und zuerst Gott zukommt, akzidentell und sekundär der Schöpfung, wie Wesenheit, Leben Intellekt, die uns nur insofern bekannt werden, als es sie in den Geschöpfen der Gattung, der Art oder der Zahl nach gibt; und diesem Sein nach kommen sie Gott nicht zu, sondern auf unendlich hervorragendere und vorzüglichere Weise. Z. B. so wenn ich sage: 'Gott ist die Wesenheit' und sogleich noch stärker verneine, indem ich sage: 'Gott ist nicht die Wesenheit', weil er nicht die Wesenheit ist, so wie sie uns der Gattung oder der Art oder der Zahl nach bekannt ist; und sodann erkläre ich: 'er ist die Wesenheit über alle Wesenheit', und ich kann nicht sagen, in welchem Maß er über alle Wesenheit ist, da der analytische Verstand auf ein Erstes nicht vordringen kann, das nicht etwas von der Wesenheit des durch die Analyse Aufgelösten und damit nicht vermischt ist; Gott ist aber nichts von Geschöpfen, sondern er ragt vorzüglich über sie alle ins Unendliche hervor. Daher wiederum bleibt unser Intellekt, wenn er so vorgeht, im Unendlichen stehen und verströmt sich in jenem."

\section{$V I$}

Eine in theologiesystematischer Hinsicht derart anspruchsvolle Erklärung des inhaltlich und sprachlich überaus schwierigen Textes der neunten Epistel des Dionysius wie die hier kurz vorgestellte, und eine Rekonstruktion, wissenschaftstheoretische Grundlegung und Entfaltung der symbolischen Theologie konnte Albertus Magnus nur aufgrund seines profunden, auf einer breiten Quellenbasis fundierten theologischen, philosophischen und naturkundlichen Wissens gelingen. Unsere skizzenhaften Ausführungen haben ansatzweise gezeigt, dass und wie er das genuine Geschäft des Theologen unter vielerlei Gesichtspunkten wahrnimmt und reflektiert, dass er auch sehr unterschiedliche theologische und nichttheologische Quellen und Traditionen, sofern diese der theologischen Wissenschaft einen hermeneutisch nützlichen Dienst erweisen, einbezieht. Man muss hervorheben, dass die nichttheologischen Wissensbestände, die der christlichen Denktradition in weiten Teilen noch fremdartig waren und ein mit ihr konkurrierendes Modell einer sich ausschließlich auf die natürliche Vernunft stützenden Weltdeutung vertraten, wie die neu bekanntwerdende Philosophie des Aristoteles und ihre späteren Interpretationen, 
sich für Alberts theologisches und philosophisches Denken als eine enorme Herausforderung und Inspirationsquelle zugleich erwiesen. Wie produktiv sie in der Theologie eingesetzt werden konnten, zeigte sich schon im Prolog des Kommentars zu der neunten Epistel des Dionysius, wo die wissenschaftstheoretischen Grundfragen der ars de symbolica theologia im Mittelpunkt stehen. Die Indienstnahme einer Vielzahl von verschieden Quellen bei der Rekonstruktion, wissenschaftlichen Grundlegung, Auslegung und Entfaltung der symbolischen Theologie hatte diese dennoch nicht zu einem Amalgam werden lassen, sondern sie sicherte ihr einen wissenschaftlichen Status und schärfte ihr eigentümliches Profil sowie trug wesentlich zu ihrer systematischen Entfaltung bei.

Will man die Quellen, die Albert in seinem Kommentar verwendet und auch explizit benennt, typologisieren, dann wird man sie in drei Gruppen von unterschiedlicher Stärke einordnen können. Es sind (1) die theologischen Quellen, (2) das philosophische und naturwissenschaftliche Wissensgut und (3) die Dichter und Grammatiker.

Den stärksten und wichtigsten Quellenkomplex stellt naturgemäß die Theologie selbst zu Verfügung. Neben der Dionysischen Vorlage des Kommentars und weiteren Schriften aus dem Corpus Dionysiacum einschließlich der darin überlieferten Scholien und Glossen fallen am meisten ins Gewicht die Allegationen aus dem Alten und dem Neuen Testament. Der Bibel kommt in Alberts Kommentar der höchstnormative Rang zu. Sie ist die primäre Quelle, die Maßgabe, das Beweismittel und auch das wichtigste Instrument der Interpretation des Dionysiustextes und der symbolischen Theologie. Ihre leitende Funktion kommt zum Ausdruck am Anfang des Kommentars im sog. Prothema, das den gesamten Inhalt der Epistel hermeneutisch aufschlüsselt und im Voraus die Kommentierungsstrategie festlegt.

Die sekundären theologischen Quellen aus der Patristik und der Scholastik zieht Albertus Magnus vor allem zur systematischen Entfaltung der symbolischen Theologie heran. Wie oben angemerkt, sieht er den Kirchenvätern als großen Verdienst an, dass sie aus der symbolischen Redeweise der Bibel und den geschilderten partikulären Sachverhalten die darin verborgenen, allgemeinen Lehrgehalte herausgearbeitet und systematisch zur Darstellung gebracht haben. ${ }^{65} \mathrm{Zu}$ den explizit zitierten Auto-

${ }^{65}$ Cf. oben Anm.37 mit den darauf folgenden Ausführungen im Textkorpus dieses Beitrags. 
Albertus Magnus über die ars de symbolica theologia des Dionysius Areopagita | 331

ritäten gehören Hieronymus, Augustinus (und Ps.-Augustinus), die Sancti Graecorum, die sich als Nemesius von Emesa und Johannes von Damaskus nachweisen lassen, Gregor der Große und Petrus Lombardus. ${ }^{66}$

Die zweitwichtigste Gruppe der Quellen des Kommentars bilden die philosophischen und naturwissenschaftlichen Schriften aus der griechischen Antike, unter denen das Corpus Aristotelicum die entscheidenden Maßstäbe vor allem hinsichtlich der Wissenschaftslehre und der Naturkenntnis setzt. Man begegnet ferner dem arabischen Aristotelesausleger Averroes (Commentator) und Pseudo-Cicero als dem Exponenten der Stoischen Mnemonik. ${ }^{67}$ Die Anknüpfung an die macrobianische Tugendlehre erfolgt unter einem undifferenzierten Verweis auf die Moralphilosophie. Über die Funktion und Bedeutung dieses Quellenkomplexes, die Albert für die symbolische Theologie zu nutzen weiß, mögen die vorausgegangenen Bemerkungen hier genügen.

Hinsichtlich der letztgenannten Gruppe von Quellen -wir bezeichneten sie mit 'Dichter und Grammatiker'-, zu der nur Horaz (De arte poetica) und der Barbarismus (i. e. Buch III der Ars grammatica des Donatus) gehören, ${ }^{68}$ fällt auf, dass Albert zwar die Rolle der Dichtung in der Philosophie, wie insgesamt in den theoretischen Wissenschaften, negativ beurteilt, aber sie als Mittel zur Findung praktischer Weisheit nicht gänzlich ausschließt. Darüber hinaus unterscheidet er, wie zuvor erwähnt, zwischen profaner Dichtung und der göttlichen Poesie der Hl. Schrift und der Theologen als dem Träger der Offenbarung. ${ }^{69}$

66 Hieronymus: Albertus Magnus, Super Dionysii Epistulas, ep. IX, p.534 v.41 (Prologus Galeatus), p.541 v.27, p.546 v.11; Augustinus: ibid. p.528 v.27, p.536 v.24, p.545 v.22 (Ps.-Aug., De spiritu et anima); Sancti Graecorum (Nemesius von Emesa und Johannes von Damaskus): ibid. p.538 v.58; Gregor der Große: ibid. p.534 v.32; Petrus Lombardus: ibid. p. 536 v.10.

${ }^{67}$ Aristoteles und/oder seine Werke (sowie die allgemeine Bezeichnung 'omnes philosophi') sind in Alberts Kommentar häufiger genannt, weshalb hier die einzelnen Stellen nicht aufgelistet werden. Averroes wird zweimal zitiert, das erste Mal allerdings nur sein Werk ohne den Autornamen: Albertus Magnus, Super Dionysii Epistulas, ep. IX, p.538 v.69-70 ('quodam tractatus de memoria et reminiscentia'), p.540 v.18 ('Commentator in II Metaphysicae'). Ps.-Cicero: ibid. p.538 v.65 ('Tullius').

68 Horaz: Albertus Magnus, Super Dionysii Epistulas, ep. IX, p.533 v.37; 'Barbarismus': ibid. p.530 v.64.

${ }^{69}$ Cf. oben die entsprechenden Passagen im Abschn. IV mit Anm.39-42. Simon (1978), 96-99. 
Wir schließen nun diese Skizze mit der Feststellung, dass Alberts ungewöhnliche theologische Kompetenz und Kreativität, die sich bei seiner Auslegung der Dionysischen Epistel manifestieren, in seiner profunden Kenntnis der primären Quellen mit ihrem näheren und weiteren geistesgeschichtlichen Kontext gründen, eine Kenntnis, die Hand in Hand mit einer stupenden Vertrautheit mit den verschiedenen philosophischen Traditionen und seinem naturwissenschaftlichen Wissen geht.

$$
\begin{gathered}
\text { ANHANG } \\
\text { Albertus Magnus, Super Dionysii Epistulam IX (ad Titum) })^{70} \\
\text {-Struktur und Inhalt- }
\end{gathered}
$$

[Prolog, 528,2-65] Materia epistulae: Ars de symbolica theologia (528,6-7)

1. Multiplicitas figurarum vel symbolorum $(528,12-13)$

1.1 multitudo symbolorum et proportio ipsorum ad nos $(528,16-17)$

1.1.1 auctor multiplicationis dictae, qui est deus (528,18-19)

1.1.2 multitudo ipsa $(528,23)$

1.1.2.1 quoad videntes, 528,25$)$

1.1.2.1.1 visio sensibilis, i. e. corporalis (528,26.30-31)

1.1.2.1.2 visio imaginabilis/imaginaria, i. e. per somnium (528,26-27.33)

1.1.2.1.3 visio intellectualis, i. e. altissimus modus contemplationis secundum statum viae $(528,27.37-38)$

1.1.2.2 multiplicatio ex parte visorum $(528,40)$

1.1.2.2.1 figuris hominis $(528,41-42)$

1.1.2.2.2 figuris ignis $(528,42)$

1.1.2.2.3 cetera quae in littera tanguntur $(528,42)$

1.2 proportio symbolorum ad nos $(528,17.44)$

2. Modus expositionis symbolorum: per inspectionem similitudinis $(528,54)$

Pars I (528,66-542,23): Generalis ars exponendi symbolica de deo dicta (cf. 528,67-529,1)

I.1 Necessitas istius artis (529.4)

I.1.1 [Dionysius] remittit ad symbolicam theologiam, (...) in qua diffusius istam artem tradidit $(529,6-8)$

70 Albertus Magnus, Super Dionysii Mysticam theologiam et Epistulas, ed. Paul Simon (Alberti Magni Opera Omnia XXXVII/2), Münster 1978, p.528-546. 
Albertus Magnus über die ars de symbolica theologia des Dionysius Areopagita | 333

I.1.1.2 ponit necessitatem artis $(529,8-9)$

I.1.1.2.1 causa necessitatis ex parte ipsorum symbolorum $(530,44)$

I.1.1.2.1.1 ponit causam necessitatis (530,47.50-66.)

I.1.1.2.1.2 manifestat per effectum (530,48.67-71)

I.1.1.2.2 causa necessitatis ex parte ipsius veritatis, quae in symbolis traditur $(530,44-45.72-531,7)$

I.1.1.3 concludit propositum (529,10; 531,65-532,2)

I.2. Generalis modus symbolicae theologiae $(532,3)$

I.2.1 significationes symbolorum cum symbolis $(532,5)$

I.2.1.1 significationes symbolorum cum symbolis, quae non important aliquem defectum (532,21-22)

I.2.1.1.1 significationes symbolorum, quibus significantur processiones divinarum personarum $(532,25-26.29-55)$

I.2.1.1.2 significationes symbolorum, quibus significantur processiones creaturarum $(532,26.56-533,5)$

I.2.1.2 significationes symbolorum cum symbolis, quae important defectum, qui est ex peccato $(532,22-23 ; 533,6-533,47)$

I.2.2 modus exponendi symbola, i. e. modus communis symbolicae theologiae, quo in corporalibus symbolis accipiuntur spiritualia et divina (532,6; $533,48-52)$

I.2.2. 1 causae istius modi $(533,52-53)$

I.2.2.1.1 causa, quare divina traduntur in sensibilibus symbolis ex parte audientium $(533,55-57 ; 534,1-25)$

I.2.2.1.1.1 proponit, quod intendit (difficultas propter occultationem: $533,61.64-70)$

I.2.2.1.1.2 reddit causam propositi (divina occulta scientia: 533,62-63; 534,1-25)

I.2.2.1.2 causa ex parte ipsius theologici negotii $(533,57-58 ; 535,10-11)$

I.2.2.1.2.1 in quo divina significantur nobis per symbola (i. e. per negationem, $535,11-12 ; 536,18)$

I.2.2.1.2.1.1 traditio secreta et mystica quantum ad ea quae ipsis occultantur $(535,13-14 ; 536,15-20)$

I.2.2.1.2.1.2 traditio significativa quantum ad symbola et perfectiva quantum ad veritatem $(535,14-15 ; 536,22-28)$

I.2.2.1.2.1.3 actionibus mysteriorum collocat in deo, i. e. in inspectionem divinae veritatis $(535,15-16 ; 537,44-49)$

I.2.2.1.2.2 in quo divina significantur nobis per propria (i. e. per affirmationem, $535,11-13 ; 536,20-22)$ 
I.2.2.1.2.2.1 traditio apparens et notior $(536,21)$

I.2.2.1.2.2.2 traditio philosophica, quia per modum philosophiae procedit, et receptiva, i. e. approbativa (536,28-31)

I.2.2.1.2.2.3 persuadet veritatem quantum ad ipsas conclusiones et ligat quantum ad conexiones argumentorum ex principiis fidei (536,39-44)

I.2.2.1.3 causa ex parte ipsius animae, quae dicit 'propter quid' respectu secundae causae [ex parte theologici negotii] (533,58-60; 536,50-52.71-73)

I.2.2.1.3.1 manifestat, quod scriptura utitur symbolis (536,53-54.56-70)

I.2.2.1.3.2 assignat causam eius $(536,54-55)$

I.2.2.1.3.2.1 ponit causam $(536,71-72 ; 537,68-538,9)$

I.2.2.1.3.2.2 probat eam $(536,72-73 ; 538,10-15)$

I.2.2.1.3.2.2.1 ex parte eorum qui sine velaminibus accipiunt divina sicut intellectuali visione $(538,15-22)$

I.2.2.1.3.2.2.2 ex ipsa divina operatione (538.13-14; 539.31-40)

I.2.2.1.3.2.2.3 ex divisione sacrae scripturae (538,14-15; 539,41-57)

I.2.2.2 modus intentus, qui ex illis causis concluditur $(533,53-54 ; 541,32)$

I.2.2.2.1 concludit, quod in symbolis sunt aliqua perscrutanda (541,32-34.36-65)

I.2.2.2.2 [concludit,] quid in eis quaerere debeamus (541,34-35.66-542,23)

\section{Quaestiones/Dubia:}

1 Non videtur, quod in statu viae possimus nudam veritatem divinam inspicere (531,8-64)

2 Videtur, quod amatores veritatis non debeant destruere huiusmodi signa symbolorum $(534,26-535,9)$

3 Dubitatur de divisionibus theologici negotii $(535,10-536,14)^{71}$

a) negotium materialiter, i. e. quantum ad ea quae dicuntur de deo $(535,58)$

a1) affirmativum negotium de divinis personis $(535,21)$

a2) affirmativum negotium de divinis nominibus $(535,22)$

a3) affirmativum negotium de symbolis $(535,22)$

a4) negativum negotium [i. e. mysticum] (535,22-23)

b) negotium formaliter, i. e. quantum ad modum dicendi aliquid de deo (535,61-62)

71 Die materielle Einteilung des theologischen Negotium gilt nicht für die symbolische Theologie, welche unter formaler Rücksicht aufgefasst und unterschieden wird (quantum ad modum dicendi aliquid de deo vel sine signis aut per signa, i. e. per affirmationem et negationem). 
b1) sine signis [i. e. per propria] $(535,63)$

b2) per signa $(535,64)$

4 Utrum hoc possit esse verum, quod oportet esse duplex theologicum negotium [i. e. per propria et per symbola] ad perfectionem utriusque partis animae, scilicet divisibilis et indivisibilis (537,1-67)

5 Videtur, quod qui accipit per intellectum divina sine symbolis, non debeat ea figuris velare $(538,23-539,30)$

6 Obiectio de modis essentialibus philosophiae (539,58-540,59)

Pars II (542,24-546-12): Responsio Dionysii ad quaestionem Titi (cf. $529,1-2)$

II.1 Exponit quaedam symbolorum de quibus Titus quaesierat (542,26-27)

II.1.1 exponit quaedam quae circumstant potum et cibum et convivium sapientiae, de quibus ille quiesierat (542,32-34)

II.1.1.1 quae circumstant alimentum in generali, sub quo continentur spiritualia alimenta (542,36-38.41-43)

II.1.1.1.1 proponit duodecim proprietates nutrimenti (542,43-44)

II.1.1.1.1.1 quae consequuntur alimentum secundum quod servit nutritive $(542,44-45)$

II.1.1.1.1.1.1 ex parte subiecti, circa quod primo accidit nutritio (542,58-60)

II.1.1.1.1.1.1.1 ex parte eius quod alitur (542,61-62)

II.1.1.1.1.1.1.1.1 quantum ad partes materiales, quae fluunt et refluunt (542,62-64)

II.1.1.1.1.1.1.1.2 quantum ad partes secundum speciem, quae permanent et ex quibus est integritas viventis (542,64-67)

II.1.1.1.1.1.1.2 ex parte alentis $(542,62)$

II.1.1.1.1.1.1.2.1 ex parte formae (542,67-70)

II.1.1.1.1.1.1.2.1 ex parte materiae (542,70-72)

II.1.1.1.1.1.2 ex parte consequentis in anima (542,60-61)

II.1.1.1.1.1.2.1 secundum habitum (542, 72-76)

II.1.1.1.1.1.2.2 secundum actum (542,76-77)

II.1.1.1.1.1.2.2.1 in affectu $(542,77)$

II.1.1.1.1.1.2.2.1.1 per remotionem a contrario (542,78-79)

II.1.1.1.1.1.2.2.1.2 quantum ad finem (542,79-80)

II.1.1.1.1.1.2.2.2 in operativa virtute $(542,77)$

II.1.1.1.1.1.2.2.2.1 per remotionem a contrario $(542,80-543,1)$ 
II.1.1.1.1.1.2.2.2.2 quantum ad finem $(543,2)$

II.1.1.1.1.2 quae consequuntur alimentum secundum quod servit augmentative $(542,46)$

II.1.1.1.1.2.1 quantum ad finem sive ad terminum ad quem (542,47-51)

II.1.1.1.1.2.2 secundum remotionem a contrario vel quantum ad terminum a quo $(542,51-53)$

II.1.1.1.1.2.2.1 quantum ad ipsam imperfectionem quantitatis (542,53-55)

II.1.1.1.1.2.2.2 quoad deperditi restaurationem (542,55-56)

II.1.1.1.1.2.2.3 quantum ad defectum actionis, qui consequitur imperfectionem quantitatis (542,56-58)

II.1.1.1.2 concludit secundum artem habitam et proprietates positas, quod divina sapientia convenienter dicitur, quod proposuit cratera et propinavit vinum, et quod ante haec proposuit fortia alimenta (543,3-13)

II.1.1.2 quae circumstant craterem (542,38-39; 543,14-25)

II.1.1.3 quae circumstant ipsam domum (542,39-40; 543,26-43)

II.1.2 exponit specialiter praedicta symbola [i. e. quid significant alimenta] $(542,34-36 ; 543,44-46)$

II.1.2.1 exponit ea, secundum quod conveniunt hominibus in statu viae $(543,46-47)$

II.1.2.1.1 proponit quaestionem (543,50-51.53-58)

II.1.2.1.2 determinat eam $(543,51-52 ; 544,1)$

II.1.2.1.2.1 quid sit [i. e. spiritualiter significat] solidum alimentum (544,1-24)

II.1.2.1.2.2 quid sit [i.e. spiritualiter significat] humidum [alimentum] (544,25-26)

II.1.2.1.2.2.1 quid significet humidum alimentum in generali $(544,26-38)$

II.1.2.1.2.2.2 quid significet humidum alimentum in speciali $(544,27.39-56)$

II.1.2.2 secundum quod conveniunt deo (543,47-48; 544,57-59)

II.1.2.2.1 quomodo ebrietas convenit secundum abundantiam (544,60-61.63-545,1)

II.1.2.2.2 quomodo [ebrietas] convenit secundum excessum (544,61-62; 545,1-8)

II.1.2.3 exponit secundum quod conveniunt angelis et hominibus in patria $(543,49-50 ; 545,9-12)$

II.1.2.3.1 in generali (545,13-14.16-26)

II.1.2.3.2 in speciali $(545,14-15.27-57)$

II.2 Excusat se ab expositione aliorum per hoc quod mittit sibi Symbolicam theologiam, quam Timotheo fecit et licentiat se ab eo $(542,28-31 ; 545,56-546,12)$ 
Albertus Magnus über die ars de symbolica theologia des Dionysius Areopagita | 337

\section{BIBLIOGR APHIE}

\section{Primärliteratur}

Albertus Magnus, De bono, ed. Heinrich Kühle et al. (Alberti Magni Opera Omnia XXVIII), Münster 1951.

$\longrightarrow$ De causis et processu universitatis a prima causa, ed. Winfried Fauser (Alberti Magni Opera Omnia XVII/2), Münster 1993.

$\longrightarrow$, De homine, eds. Henryk Anzulewicz/Joachim R. Söder (Alberti Magni Opera Omnia XXVII/2), Münster 2008.

$\longrightarrow$, De natura boni, ed. Ephrem Filthaut (Alberti Magni Opera Omnia XXV/1), Münster 1974.

$\longrightarrow$, De IV coaequaevis (Summa de creaturis I), ed. Steph. Caes. Aug. Borgnet (Alberti Magni Opera Omnia XXXIV), Paris 1895.

$\longrightarrow$, De sacramentis, ed. Albert Ohlmeyer (Alberti Magni Opera Omnia XXVI), Münster 1958.

— De somno et vigilia, ed. Aug. Borgnet (Alberti Magni Opera Omnia IX), Paris 1890, 121-207.

— Ethica, ed. Aug. Borgnet (Alberti Magni Opera Omnia VII), Paris 1891.

$\longrightarrow$, In I Sententiarum, dist.I-XXV, ed. Aug. Borgnet (Alberti Magni Opera Omnia XXV), Paris 1893.

—, Metaphysica I-V,ed. Bernhard Geyer (Alberti Magni Opera Omnia XVI/1), Münster 1960.

—, Summa theologiae I, ed. Dionysius Siedler et al. (Alberti Magni Opera Omnia XXXIV/1), Münster 1978.

—, Super Dionysii Mysticam theologiam et Epistulas, ed. Paul Simon (Alberti Magni Opera Omnia XXXVII/2), Münster 1978 (Epistula septima: p.501-513; Epistula nona: p.528-546).

—, Super Dionysium De caelesti hierarchia, eds. Paul Simon/Wilhelm Kübel (Alberti Magni Opera Omnia XXXVI/1), Münster 1993.

—, Super Ethica VI-X, ed. Wilhelm Kübel (Alberti Magni Opera Omnia XIV/2), Münster 1987.

—, Super Matthaeum I-IX, ed. Bernhard Schmidt (Alberti Magni Opera Omnia XXI/1), Münster 1987.

Aristoteles, De generatione animalium; De divinatione per somnum; De insomniis; Ethica Nicomachea, in Aristoteles Graece, ex recensione Immanuelis Bekkeri ed. Academia Regia Borussica, Berlin 1831. 
Dionysiaca. Recueil donnant l'ensemble des traductions latines des ouvrages attribués au Denys de l'Aréopage, tome I, ed. Philippe Chevallier, Bruges 1937.

Dionysius Areopagita (Pseudo-), Epistola IX, ed. Jacques-Paul Migne (Patrologia Graeca 3), Paris 1889, 1103-1118.

— Epistula 9, ed. Adolf M. Ritter (Corpus Dionysiacum II. Patristische Studien und Texte 36), Berlin/New York 1991, 193-207.

—., Epistula nona, in: Albertus Magnus, Super Dionysii Mysticam theologiam et Epistulas, ep. IX, p.528 v.68-76, p.531 v.67-75, p.532 v.87-89, p.533 v.71-82, p. 535 v.84-87, p. 536 v.74-80, p. 538 v.74-80, p. 541 v. $71-80$, p. 542 v.81-88, p. 543 v.59-78, p.544 v.71-81, p.545 v.69-81.

\section{Sekundärliteratur}

Anzulewicz, Henryk (1999), Die theologische Relevanz des Bildbegriffs und des Spiegelbildmodells in den Frübwerken des Albertus Magnus (Beiträge zur Geschichte der Philosophie und Theologie des Mittelalters NF 53/II), Münster.

—, (2000), "Pseudo-Dionysius Areopagita und das Strukturprinzip des Denkens von Albert dem Großen", in Die Dionysius-Rezeption im Mittelalter, eds. Tzotcho Boiadjiev et al. (Rencontres de Philosophie Médiévale 9), Turnhout, 251-295.

—, (2002), "Ab eodem in idem. Verständnis und hermeneutische Funktion der Kreislaufformel bei Albertus Magnus", in Chemins de la pensée médiévale. Études offertes à Zénon Kalura, eds. Paul J.J.M. Bakker et al. (Textes et Études du Moyen Âge 20), Turnhout, 323-350.

_ (2007), "Zwischen Faszination und Ablehnung: Theologie und Philosophie im 13. Jh. in ihrem Verhältnis zueinander", in What is «Theology» in the Middle Ages?, ed. Mikołaj Olszewski (Archa Verbi. Subsidia I), Münster, 129-165.

__, (2008), "Albertus Magnus und die moderne Kunst", Archiv für mittelalterliche Philosophie und Kultur 14, 28-45.

, (2009), "Die Emanationslehre des Albertus Magnus: Genese, Gestalt und Bedeutung", in Via Alberti. Texte - Quellen - Interpretationen, eds. Ludger Honnefelder et al. (Subsidia Albertina II), Münster, 219-242.

_-, (im Druck I), "The Systematic Theology of Albert the Great", in The Universal Doctor: Albertus Magnus on Theology, Philosophy, and the Sciences, ed. Irven M. Resnick, Turnhout.

-, (im Druck II), "Albert the Great", in The Cambridge History of Medieval Theology, 1050-15000, eds. Joseph Wawrykow/Thomas J. Prügl, Cambridge. 
Albertus Magnus über die ars de symbolica theologia des Dionysius Areopagita | 339

Anzulewicz, Henryk/Rigo, Caterina (2002), "Reductio ad esse divinum. Zur Vollendung des Menschen nach Albertus Magnus", in Ende und Vollendung. Eschatologische Perspektiven im Mittelalter, eds. Jan A. Aertsen /Martin Pickavé (Miscellanea Mediaevalia 29), Berlin/New York, 388-416.

Beierwaltes, Werner (1994), Eriugena. Grundzüge seines Denkens, Frankfurt am Main.

(1997), "Dionysios Areopagites - ein christlicher Proklos?", in Platon in der abendländischen Geistesgeschichte, eds. Theo Kobusch/Burkhard Mojsisch, Darmstadt, 71-100.

Burger, Maria (1998), "Das Verhältnis von Philosophie und Theologie in den Dionysius-Kommentaren Alberts des Großen", in Was ist Philosophie im Mittelalter?, eds. Jan A. Aertsen/Andreas Speer (Miscellanea Mediaevalia 26), Berlin/New York, 579-586.

Dondaine Hiacinthe-Françoise (1952), 'L’objet et le 'medium' de la vision béatifique chez les théologiens du XIII" siècle", Recherches de Théologie ancienne et médiévale 19, 60-130.

Grabmann, Martin (1933; Repr. 1983), Die Geschichte der katholischen Theologie seit dem Ausgang der Väterzeit, Freiburg im Breisgau (Repr. Darmstadt).

Enders, Markus (2007), "Zur Bedeutung des Ausdrucks theologia im 12. Jahrhundert und seinen antiken Quellen", in What is «Theology» in the Middle Ages?, ed. Mikołaj Olszewski (Archa Verbi. Subsidia I), Münster, 19-37.

Hödl, Ludwig (1994), "Opus naturae est opus intelligentiae. Ein neuplatonisches Axiom im aristotelischen Verständnis des Albertus Magnus", in Averroismus im Mittelalter und in der Renaissance, eds. Friedrich Niewöhner/Loris Sturlese, 132-148.

Jordan, Mark D. (1992), "Albert the Great and the Hierarchy of Sciences", Faith and Philosophy 9, 483-499.

Kapriev, Georgi (2005), Philosophie in Byzanz, Würzburg.

Koch, Josef (1956-1957; Repr. 1973), "Augustinischer und Dionysischer Neuplatonismus und das Mittelalter", Kant-Studien 48/2, 117-133 (Repr.: id., Kleine Schriften, I [Storia e Letteratura 127], 3-25).

Leinsle, Ulrich G. (1995), Einführung in die scholastische Theologie (Uni-Taschenbücher 1865), Paderborn/München/Wien/Zürich.

Libera, Alain de (1989), "Philosophie et Théologie chez Albert le Grand et dans l'école dominicaine allemande", in Die Kölner Universität im Mittelalter, eds. Albert Zimmermann/Gudrun Vuillemin-Diem (Miscellanea Mediaevalia 20), Berlin/New York, 49-67.

Lucentini, Paolo (1987), "L'eresia di Amalrico", in Eringena redivivus. Zur Wirkungsgeschichte seines Denkens im Mittelalter und im Übergang zur Neuzeit, ed. Werner 
Beierwaltes (Abhandlungen der Heidelberger Akademie der Wissenschaften. Philos.-hist. Klasse, Jahrgang 1987, 1. Abhandlung), Heidelberg, 174-191.

Makris, Georgios (2000), "Zwischen Hypatios von Ephesos und Lorenzo Valla. Die Areopagitische Echtheitsfrage im Mittelalter", in Die Dionysius-Rezeption im Mittelalter, eds. Tzotcho Boiadjiev et al. (Rencontres de Philosophie Médiévale 9), Turnhout, 3-39.

Meis, Anneliese (2006), "El misterio de la alteridad en Alberto Magno Super Mysticam Theologiam Dionysii", Teología y Vida 47/4, 541-574.

___, (2008), "Introducción", in Alberto Magno. Sobre la Teología Mística de Dionisio/Super Mysticam Theologiam Dionysii. Según el texto de la Editio Coloniensis traducido y editado por Anneliese Meis. Latín-español (Anales de la Facultad de Teología LIX/2008, Cuaderno 1/2), Santiago: Pontificia Universidad Católica de Chile, 15-36.

Merle, Hélène (1986), “Ars”, Bulletin de Philosophie Médiévale 28, 95-133.

Möhle, Hannes (2008), “Zum Verhältnis von Theologie und Philosophie bei Albert dem Großen”, in Rheinisch - Kölnisch - Katholisch. Beiträge zur Kirchen- und Landesgeschichte sowie zur Geschichte des Buch- und Bibliothekswesens der Rheinlande, eds. Siegfried Schmidt et al. (Libelli Rhenani 25), 147-162.

Müller, Jörn (2001), Natürliche Moral und philosophische Ethik bei Albertus Magnus (Beiträge zur Geschichte der Philosophie und Theologie des Mittelalters NF 59), Münster.

Rodolfi, Anna (2005), "Il ruolo delle immagini sensibili nella dottrina della conoscenza profetica di Alberto Magno", Annali del Dipartimento di Filosofia. Università degli Studi di Firenze XI, 79-107.

Saffrey, Henri Dominique (1996), "Les débuts de la théologie comme science (III'-VI" siècle)", Revue des Sciences philosophiques et théologiques 80, 201-220.

Schlosser, Marianne (2000), Lucerna in caliginoso loco. Aspekte des Prophetie-Begriffes in der scholastischen Theologie (Münchener Universitätsschriften. Kath.-Theol. Fakultät. Veröffentlichungen des Grabmann-Institutes NF 43), Paderborn.

Senner, Walter (2009), Alberts des Großen Verständnis von Theologie und Philosophie (Lectio Albertina 9), Münster.

Sicouly, Pablo C. (2002), "Filosofía y Teología en San Alberto Magno, comentador del Corpus Dionysiacum. Nuevas perspectivas a la luz de algunos estudios recientes", Studium. Filosofía y Teología, 5/9 (Buenos Aires/Tucumán) 23-37.

—, (2003), "Prima philosophia y theologia en san Alberto Magno", Fe y razón; encuentro y mediación (Jornadas de Estudio sobre el pensamiento Patrístico y Medieval, UNSTA 2002), Tucumán, 109-123 
Simon, Paul (1972), "Prolegomena", in: Albertus Magnus, Super Dionysium De divinis nominibus, ed. Paul Simon (Alberti Magni Opera Omnia XXXVII/1), Münster, V-XX.

—, (1978), "Albertus Magnus und die Dichter", in Xenia Medii Aevi historiam illustrantia oblata Thomae Kaeppeli O.P. (Storia e Letteratura 141), Roma, 85-99.

Suchla, Beate R. (1998; ${ }^{2}$ 1999), "Dionysius Areopagita", in Lexikon der antiken christlichen Literatur, eds. Siegmar Döpp, Wilhelm Geerlings et al., Freiburg/ Basel/Wien, 174-177.

Takahashi, Adam (2008), "Nature, Formative Power and Intellect in the Natural Philosophy of Albert the Great", Early Science and Medicine 13, 451-481.

Wéber, Édouard (1980), "La relation de la philosophie et de la théologie selon Albert le Grand", Archives de Philosophie 43, 559-588.

Weisheipl, James A. (1980), "The Axiom opus naturae est opus intelligentiae and its Origins", in: Albertus Magnus Doctor universalis 1280/1980, eds. Gerbert Meyer et al. (Walberberger Studien. Philosophische Reihe 6), Mainz, 441-463.

Weismantel, Tobias (2008/2009), Ars nominandi Deum. Die Ontosemantik der Gottesprädikate in den Dionysiuskommentaren des Albertus Magnus, ungedruckte Diss. Universität Regensburg, Kath.-Theol. Fakultät. 
Resumen: Alberto Magno se cuenta entre los más importantes receptores e intérpretes escolásticos de la totalidad del Corpus Dionysiacum. Se puede constatar ya en su primera obra De natura boni y en sus obras teológicas sistemáticas tempranas un interés especial por la teología neoplatónica del Areopagita y la influencia creciente de ella sobre su comprensión de teología y su forma de pensar. En este estudio se esboza la comprensión de Alberto respecto de la teología simbólica, que él desarrolla en su Comentario de la carta novena de Dionisio Areopagita. Se describe en grandes líneas, cómo Alberto, siguiendo la epístola dionisiana, por un lado reconstruye la doctrina no conservada y tal vez no escrita sobre la teología mística del supuesto discípulo del Apóstol, y por otro lado la fundamenta de modo científico-teórico y la desarrolla sistemáticamente. Primero se enfoca la comprensión de la teología de Alberto y su doctrina teológica de la ciencia contenida en el Comentario de las sentencias (libro 1) y su significado científico-teórico epocal. Luego siguen las explicaciones del modo albertino de proceder en la explicación de la epístola de Dionisio y su concepto de arte (ars), que aplica a la teología simbólica. Los párrafos siguientes del estudio se dedican a los presupuestos objetivos y hermenéuticos del prólogo del Comentario de Alberto, a la presentación de su propia comprensión de la teología simbólica, que formula según la idea central de la carta, así como también a su comprensión de la identidad y la diferencia de la teología simbólica y mística. El estudio concluye con observaciones críticas a la interpretación de Alberto de las fuentes de la epístola dionisiana y con la valoración de su competencia y creatividad teológica y filosófica. En el anexo se presenta la estructura y el contenido del Comentario de la epístola novena de Dionisio en su reconstrucción sobre la base de las indicaciones del autor.

Palabras clave: Alberto Magno, Dionisio Areopagita, symbolica theologia, mystica theologia, teoria de la ciencia teológica.

Abstract. Albertus Magnus is regarded as one of the most important high scholastic recipients and interpreters of the complete Corpus Dionysiacum. In his first work, De natura boni, and in his early systematic theological works one may already identify a special interest in the Areopagite's neoplatonic theology and its increasing influence on Albert's understanding of theology and his way of thinking. In this article Albert's conception of symbolic theology which he presented in his commentary on the ninth letter of Dionysius the Areopagite is delineated. A general outline is given of the fact that, and the way in which, relying on Dionysius's epistle, he reconstructs the Apostle's supposed pupil's doctrine of mystical theology, which was not preserved or rather not written, and on the other hand justifies it with theoretical rigour and develops it in a systematic way. First, Albert's understanding of theology and his theological system (Wissenschaftslebre) from the Commentary on the Sentences (book I) as well as its epoch-making significance for the history of thought will come under consideration. This is followed by a discussion of his procedure in interpreting the epistle of Dionysius and of the concept of artistry (ars) 
which he applies to symbolic theology. The following sections of the investigation are dedicated to the substantial and hermeneutic guidelines of the prologue of Albert's commentary, to the presentation of his own conception of symbolic theology which he formulates on the basis of the letter, and also to his understanding of the identity and difference of symbolic and mystic theology. The investigation concludes with comments on the assessment of the sources for Albert's interpretation of Dionysius's epistle and with an evaluation of his theological and philosophical competence and creativity. In the appendix the structure and content of the commentary on the ninth epistle of Dionysius are presented in a reconstruction that is based on the author's indications.

Keywords: Albertus Magnus, Dionysius the Areopagite, symbolic theology, mystic theology, concept of theology (theologische Wissenschaftslehre). 